\title{
The interplay between ambipolar electric field and Coulomb collisions in the solar wind acceleration region
}

\author{
L. Berčič ${ }^{1,2}$, S. Landi ${ }^{1,3}$, M. Maksimović ${ }^{2}$ \\ ${ }^{1}$ Physics and Astronomy Department, University of Florence, Firenze, Italy \\ ${ }^{2}$ LESIA, Observatoire de Paris, PSL Research University, CNRS, UPMC Université Paris 6, Université \\ Paris-Diderot, Meudon, France \\ ${ }^{3}$ INAF - Osservatorio Astrofisico di Arcetri, Firenze, Italy
}

\section{Key Points:}

- We use a kinetic model of expanding solar wind accounting for Coulomb collisions. This model produces a slow, supersonic solar wind proton population accelerated only through the ambipolar electric field, which arises due to the difference of mass between electron and proton.

- The self-consistently calculated ambipolar electric field in the model is on the order of Dreicer electric field.

- We present the radial evolution of the strahl electron component under the influence of Coulomb collisions.

Corresponding author: Laura Berčič, laura.bercic@obspm.fr 


The results presented in this work were obtained using a numerical kinetic model of the solar wind expansion accounting for Coulomb collisions (Landi \& Pantellini, 2001, 2003; Landi et al., 2010, 2012, 2014). The model does not capture all of the solar wind physics, but instead allows a detailed view into a kinetic behaviour of the colliding solar wind electrons in the near-Sun regions. In comparison to the existing exospheric models, the benefits of the numerical model are:

- a statistical treatment of binary Coulomb collisions instead of using a FokkerPlanck collision operator,

- a self-consistent calculation of the ambipolar electric field, and

- a continuous transition between the collisional and collision-less regime (the exobase is not defined as a single radial distance and is not required as an input parameter).

The modelled solar wind and its evolution through the acceleration region is described with plasma moments in Sec. 3. The analysis of the obtained electron VDFs permits an investigation of the effects of the ambipolar electric field on the VDFs (Sec. 4), and of the radial evolution of the strahl electron component (Sec. 5).

\section{Numerical model}

We use the fully kinetic model BiCoP (Binary Collisions in Plasmas) to simulate the radial expansion of the solar wind. Details of the model are described by Landi and Pantellini $(2001,2003)$, who in the first work present the evolution of solar wind moments over the first $0.2 \mathrm{R}_{S}$ above the solar surface. In the second work they extend their simulation domain to reach up to $50 \mathrm{R}_{S}$, however, with decreased proton to electron mass ratio. Later works with $\mathrm{BiCoP}$ use realistic solar wind characteristics, like proton-electron mass ratio and the input plasma moments, and present the radial evolution of electron VDF between 0.3 and $3 \mathrm{R}_{S}$, where the solar wind has already reached its terminal velocity and the effect of gravity can be neglected (Landi et al., 2012 , 2014). They show that the model produces a two-component electron VDF function - consisting of the core and the strahl, and the global solar wind moments which compare well with the observed values. With the evolution of the code as well as computer technology we are now able to conduct the simulations of the solar wind acceleration region where the effect of gravity is of great importance $\left(1 R_{S}-49 R_{S}\right)$ using real proton to mass ratio and reproducing the plasma moments measured by the Parker Solar Probe (Fox et al., 2016).

A schematics of the simulation setup is shown in Fig. 1. The model is 1dimensional in space and 3-dimensional in velocity space. $N$ macroparticles are included in the simulations representing two species - electrons and protons, defined by their opposite signed charge and realistic mass ratio $\left(\frac{m_{p}}{m_{e}}=1837\right)$. The particles are accelerated by the Sun's gravitational force and the ambipolar electric field force:

$$
\frac{d^{2} r}{d t^{2}}=-\frac{G M_{S}}{r^{2}}+\frac{\vec{L}^{2}}{m_{i}^{2} r^{3}}+\frac{q}{m_{i}} E(r),
$$

where $r$ is the radial distance from the Sun, $G$ the gravitational constant, $M_{S}$ the mass of the Sun, $m_{i}$ the mass of a particle and $E(r)$ the ambipolar electric field. $\vec{L}$ is the angular momentum that can be expressed in terms of perpendicular particle velocity: $\vec{L}=m_{i} \vec{r} \times \vec{v}$. In the model we assume a radial magnetic field so that angular magnetic conservation is equivalent to the magnetic moment conservation (Landi et al., 2012).

The main parameter defining the behaviour of the system is the ratio between the gravitational potential and the electron thermal energy at $r_{0}$, the distance from 


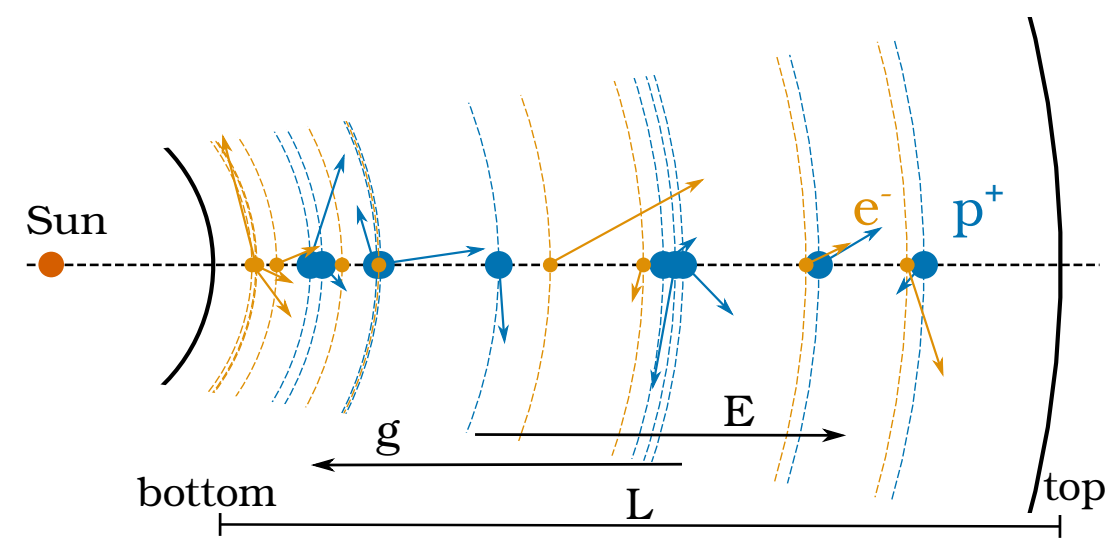

the Sun's centre and the simulation bottom boundary:

$$
\gamma=\frac{G M_{S}}{r_{0}} \cdot \frac{m_{e}}{2 k_{B} T_{e, b o t}},
$$

where $T_{e, b o t}$ is the temperature of electrons at the bottom simulation boundary. Gravity is thus expressed as

$$
g_{0}=\gamma \frac{l}{r_{0}},
$$

with $l$ the length of the simulation domain.

A benefit of the described kinetic model is a self-consistent calculation of the ambipolar electric field. The electric field in the simulation is composed of two contributions. First is a global electric field, radially decreasing with $r^{2}$, keeping the balance between electron and proton fluxes. Second is the charge-neutralising electric field, a local polarisation field resulting from local charge imbalances (Landi \& Pantellini, 2001). This field is obtained by considering each particle as a thin spherical conducting shell centred in the Sun, and calculating the local field of a system of conducting spherical plates (Landi \& Pantellini, 2003).

Another BiCoP strength is the statistical treatment of binary Coulomb collisions. When two particles find themselves on the same position along the dimension of the simulation, they can either suffer an elastic collision or pass each other undisturbed. The collision probability decreases with $v^{4}$, as predicted by Coulomb cross-section. To save the computational time particles with relative velocity lower than a defined velocity limit $\left(v_{C}\right)$ will collide every time. Landi and Pantellini (2001) show that this computational simplification does not change the Coulomb collisions properties and have the same effect on the electron VDF as long as $v_{C}$ is smaller than the thermal velocity of the electrons at any radial distance $\left(v_{C}<v_{t h}\right)$. Even more, we make use of this parameter to vary the collisionality of the system.

The one-dimensional simulation domain is limited by the bottom and the top boundary, of which the bottom boundary is located closer to the Sun. The shape of the proton and electron VDFs in these two points is defined with the input parameters $T_{e, p, b o t}, T_{e, t o p}$. In the present study all the boundary VDFs are isotropic 
Table 1. Presented simulation runs and their crucial input parameters.

\begin{tabular}{cc|cccc}
\hline Parameters & Unit & $\mathrm{A}$ & $\mathrm{LC}$ & $\mathrm{MC}$ & $\mathrm{HC}$ \\
\hline $\mathrm{N}$ & & 22500 & 22500 & 22500 & 22500 \\
$\mathrm{v}_{C}$ & $v_{t h, 0}$ & 0.4 & $\mathbf{0 . 4}$ & $\mathbf{0 . 3}$ & $\mathbf{0 . 2}$ \\
$\mathrm{T}_{e, p, \text { bot }}$ & $10^{6} \mathrm{~K}$ & 2 & 1.4 & 1.4 & 1.4 \\
$\mathrm{~T}_{e, t o p}$ & $10^{6} \mathrm{~K}$ & 0.82 & 0.77 & 0.77 & 0.77 \\
$\mathrm{~g}_{0}$ & & 0.1416 & 0.0225 & 0.0225 & 0.0225 \\
$\mathrm{r}$ & $\mathrm{R}_{S}$ & $\mathbf{1 - 4 6}$ & $3-49$ & $3-49$ & $3-49$ \\
$\mathrm{v}_{\text {bot }}$ & $\mathrm{km} / \mathrm{s}$ & 0 & 104 & 104 & 104 \\
$\mathrm{v}_{\text {top }}$ & $\mathrm{km} / \mathrm{s}$ & 218 & 228 & 228 & 228 \\
\hline
\end{tabular}

and Maxwellian-like, which leaves us with the temperature and the bulk velocity as the only free parameters. The bottom and top velocities are the same for both species $\left(v_{b o t}, v_{t o p}\right)$. We define the temperature of the both species at the bottom $\left(T_{e, b o t}, T_{p, b o t}\right)$, and the temperature of electrons on the top $\left(T_{e, t o p}\right)$, as the protons at the top have a supersonic velocity, thus all leaving the simulation domain and being re-injected at the bottom. On the contrary, electrons are subsonic, thus a portion of them has to be injected back from the top boundary with a probability and velocity which are given by the distribution function assumed at the top. The equal flux between the two species is assured everywhere in the system only by the self-consistent electric field. The kinetic model tends toward a stationary, quasi-neutral solar wind solution only if the boundary conditions are also a part of this solution. Therefore the choice of $T_{e, t o p}$ and $v_{t o p}$ is not really free, and depends on the $T_{e, b o t}$ and $T_{p, b o t}$, as well as on the collisionality of the system. For each of the presented simulation runs, test runs were preformed iterating towards good values for the top boundary parameters.

The particle's velocity distribution functions are built by binning the spatial domain in 40 bins and the velocity space in $80 \times 80$ bins in the radial and perpendicular direction. Once the stationary state has been reached the position and velocity of the particles are regularly sampled to build the velocity distribution function as function of the distance. Moments of the distribution function are also directly computed in the simulation.

The presented simulation runs with their key parameters are listed in Tab. 1.

\section{Density, velocity \& temperature}

\subsection{Method}

\subsubsection{Physical unit density}

Fig. 2 shows the radial evolution of density $(n)$, velocity $(v)$, and core electron temperature $\left(T_{e, \text { core }}\right)$ over the simulation domain for the four presented simulation runs. The physical units of the parameters in the equation of motion (Eq. 1: r, v, $\mathrm{T}, \mathrm{E})$ are all determined through the mass, gravity and temperature of the corona. Particle density, however, does not affect gravitational and electric fields, but it plays an important role for the properties of Coulomb collisions. The physical units for density are thus determined using the electron-proton collision frequency $\left(\nu_{e, p}(r)\right)$ measured in the simulation and comparing it to the Fokker-Planck electron-proton transport collision frequency for a plasma with known density $(n)$ and temperature $(T)$ : 

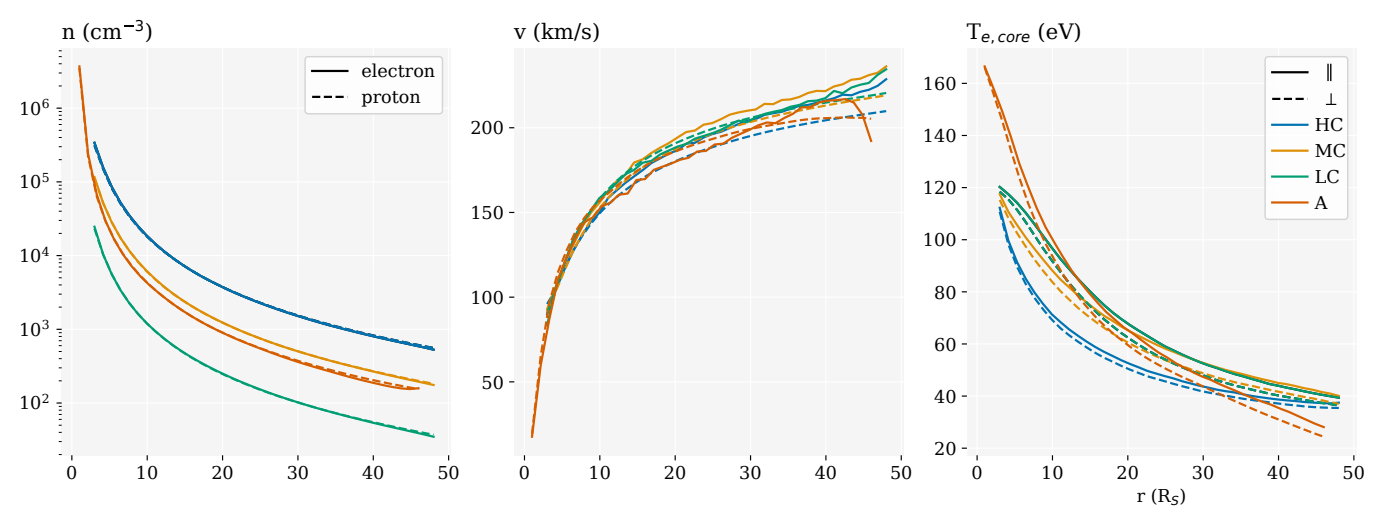

Figure 2. The evolution of electron and proton density (left), velocity (middle), and electron core parallel and perpendicular temperature (right) for all the presented simulations runs specified in Tab. 1.

$$
n=\frac{\nu_{e, p} v_{t h, 0}}{l} \cdot \frac{3 \epsilon_{0}^{2} m_{e}^{1 / 2}\left(k_{B} T\right)^{3 / 2}}{4(2 \pi)^{1 / 2} e^{4}} \frac{1}{\ln \Lambda}
$$

where $v_{t h, 0}$ is the electron thermal velocity in the first radial bin and $\ln \Lambda$ is the Coulomb logarithm:

$$
\ln \Lambda=\ln \left(\frac{12 \pi\left(\epsilon_{0} k_{B} T\right)^{3 / 2}}{n^{1 / 2} e^{3}}\right)
$$

Since the unknown density $n$ is required for the calculation of $\ln \Lambda$, we first obtain $n^{\prime}$ assuming $\ln \Lambda=24 \mathrm{in}$ Eq. 4, which is close to expected value for resulting plasma parameters: $\ln \Lambda\left(T=172 \mathrm{eV}, n=10^{6} \mathrm{~cm}^{-3}\right)=24.3, \ln \Lambda\left(T=120 \mathrm{eV}, n=10^{4} \mathrm{~cm}^{-3}\right)=$ 26.1. The final density $n_{0}$ is ten obtained by:

$$
n_{0}=n^{\prime} \frac{24}{\ln \Lambda\left(n^{\prime}\right)},
$$

The first radial bin is the densest and most collisional, thus $n_{0}$ is calculated there, and used to normalise the other radial bins accordingly with the number of particles they contain.

Simulation run A, the only presented run starting from $r_{0}=1 R_{S}$, exhibits very strong gradients in density, velocity and temperature for its first three radial bins $\left(<3 R_{S}\right.$, see Fig. 2). The Knudsen number, rises from $\sim 10^{-2}$ (1st bin) to $\sim 0.5$ (3rd bin), remaining in the collisional regime. Because the collisionality continues to stay high in the 3rd radial bin, the density there can be determined through the comparison with the Fokker-Planck collision frequency as well. However, the value obtained this way turns out to be an order of magnitude lower than the value calculated through normalisation to the first radial bin. This gives us a high uncertainty on the calculated physical unit density. The accuracy could be improved by increasing the amount of particles used in the simulation, which would substantially increase the computation time. Instead, we decided to exclude the high-gradient region just above the solar surface and conducted our other presented simulation runs staring from $r_{0}=3 R_{S}$. This way, the used amount of particles is sufficient to provide a good estimate of the physical unit density. 

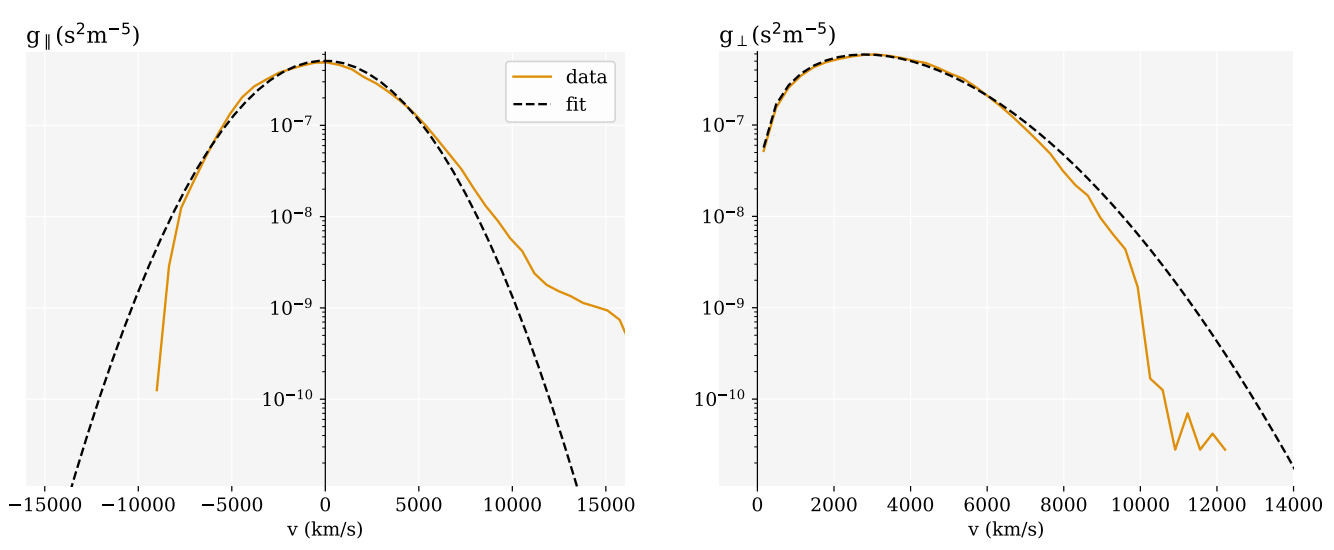

Figure 3. An example of a core fit to $g\left(v_{\|}, v_{\perp}\right)$, shown with the parallel (left), and the perpendicular (right) cut through electron VDF multiplied by $v_{\perp}$. An example is taken from simulation run $\mathrm{MC}$ at the radial distance of $35 R_{S}$.

\subsubsection{Core electron fit}

Electron VDFs in the simulation are produced for each of the 40 radial bins, on a 2-dimensional cartesian grid $(80,80)$ with a maximum velocity of $4 v_{t h, 0}$. The output function $g\left(v_{\|}, v_{\perp}\right)$ is given in a form:

$$
g\left(v_{\|}, v_{\perp}\right)=f\left(v_{\|}, v_{\perp}\right) \cdot v_{\perp}
$$

where $f\left(v_{\|}, v_{\perp}\right)$ is the velocity distribution function, and $v_{\|}$and $v_{\perp}$ are the velocities parallel and perpendicular to the magnetic field (which is in the simulations purely radial). The lower energy part of $g\left(v_{\|}, v_{\perp}\right)$ is fitted with a bi-Maxwellian distribution function multiplied by $v_{\perp}$ (see Fig. 3 ):

$$
g_{c}\left(v_{\perp}, v_{\|}\right)=A_{c} \exp \left(\frac{v_{\perp}^{2}}{w_{\perp}^{2}}+\frac{\left(v_{\|}-\Delta v_{\|}\right)^{2}}{w_{\|}^{2}}\right) \cdot v_{\perp},
$$

where $\Delta v_{\|}$is the drift velocity along the magnetic field, and the core density $\left(n_{c}\right)$, and the core parallel and perpendicular temperatures can be obtained by:

$$
n_{c}=A_{c} \cdot \pi^{3 / 2} w_{\perp}^{2} w_{\|},
$$

$$
T_{c \perp, \|}=\frac{m_{e} w_{\perp, \|}^{2}}{2 k_{B}} .
$$

\subsection{Results}

Simulation run A starts at the solar surface where we set the input proton and electron VDFs to be isotropic Maxwellians with a temperature of $2 \mathrm{MK}(172 \mathrm{eV})$ and zero bulk velocity (see Tab. 1). The density in the first radial bin reaches 4 . $10^{6} \mathrm{~cm}^{-3}$ (see Fig. 2). The density and velocity of both species are aligned verifying charge neutrality and mass flux conservation. Solar wind protons become supersonic at the distance of $4 R_{S}$ and reach their highest velocity of $206 \mathrm{~km} / \mathrm{s}$ at $42 R_{S}$. As mentioned in the previous section, due to high gradients in the first few radial bins we have a large uncertainty on the calculated density for the simulation run A. We show this run to prove that $\mathrm{BiCoP}$ can produce a supersonic wind from a static hot solar 
Table 2. Electron moments for simulations HC, MC, and LC at $35 R_{S}$.

\begin{tabular}{l|ccc}
\hline Moments & HC & MC & LC \\
\hline $\mathrm{n}\left(\mathrm{cm}^{-3}\right)$ & 1129 & 376 & 76 \\
$\mathrm{v}(\mathrm{km} / \mathrm{s})$ & 211 & 217 & 212 \\
$\mathrm{~T}_{e, c o r e, \|}(\mathrm{eV})$ & 40.7 & 48.4 & 47.6 \\
$\mathrm{~T}_{e, \text { core }, \perp}(\mathrm{eV})$ & 39.0 & 44.6 & 43.3
\end{tabular}

corona, and use the obtained temperature and velocity as a guidance for the input parameters for the runs $\mathrm{HC}$ (high collisionality), MC (medium collisionality) and LC (low collisionality) starting from $3 R_{S}$. As mentioned above, $T_{e \& p, b o t}$ and $v_{b o t}$ are not independent parameters, and a simulation starting with $T_{e \& p, b o t}=150 \mathrm{eV}$, and $v_{\text {bot }}=90 \mathrm{~km} / \mathrm{s}$ at $3 R_{S}$, as follows from the simulation run A, does not result in a stationary solution. That is because the bottom boundary proton and electron VDFs (at $3 R_{S}$ ) are set to be isotropic Maxwellians, however, in the simulation run $\mathrm{A}$ at this distance the VDFs are already deformed: protons appear anisotropic and electrons start to form a tenuous strahl population. Instead of changing the shape of the VDFs at the bottom boundary of the simulations starting at $3 R_{S}$ we decrease $T_{e \& p, b o t}$ (to $120 \mathrm{eV}$ ). This way the radial evolution of $v$ is similar for all runs, while there are some differences in the radial evolution of $T$.

Because the highest gradients are avoided for the runs $\mathrm{HC}, \mathrm{MC}$, and $\mathrm{HC}$, the used amount of particles (22500 electrons and 22500 protons) provides us with much better statistics. We study the effect of Coulomb collisions by varying the system collisionality using the input variable $v_{C}$. Run HC is the most collisional $\left(v_{C}=0.4\right)$, which is reflected in higher density and steeper decrease in core electron temperature with radial distance (see Fig. 2). The core stays close to isotropic all through the simulation domain, while in less collisional runs $\mathrm{MC}\left(v_{C}=0.3\right)$ and $\mathrm{LC}\left(v_{C}=0.2\right)$, the parallel core electron temperature is notably larger than the perpendicular one. The collisionality does not appear to have an effect on the final solar wind velocity, which is similar for all three runs, $\sim 220 \mathrm{~km} / \mathrm{s}$. This result is in contradiction with the simulation results shown by Landi and Pantellini (2003), who found that denser solar wind is accelerated to higher velocities. The discrepancy between the two results could be a consequence of the reduced proton to electron mass ratio, or much smaller amount of particles used in the simulation runs from Landi and Pantellini (2003).

For a quantitative comparison of the obtained electron moments with the Parker Solar Probe data we list the simulation values at $35 R_{S}$ in Tab. 2 .

\section{Electric field \& electric potential}

\subsection{Method}

Another simulation output is the ambipolar electric field $(E)$ at the position of every simulation particle. These values are then binned accordingly with the 40 radial bins and integrated over radial distance to obtain the electric potential $(\phi)$.

In the exospheric solar wind models, the total electric potential difference between any given distance and infinity has an important effect on the electron VDF. At any radial distance $(r)$ the antisunward moving electrons with the energy higher than the electric potential energy $\left(\mathcal{E}_{\phi}(r)\right)$ are able to escape and form the strahl population, while electrons with energy below $\mathcal{E}_{\phi}(r)$ can not escape and form a ballistic, core population. The antisunward core electrons are trapped in a potential well: they 


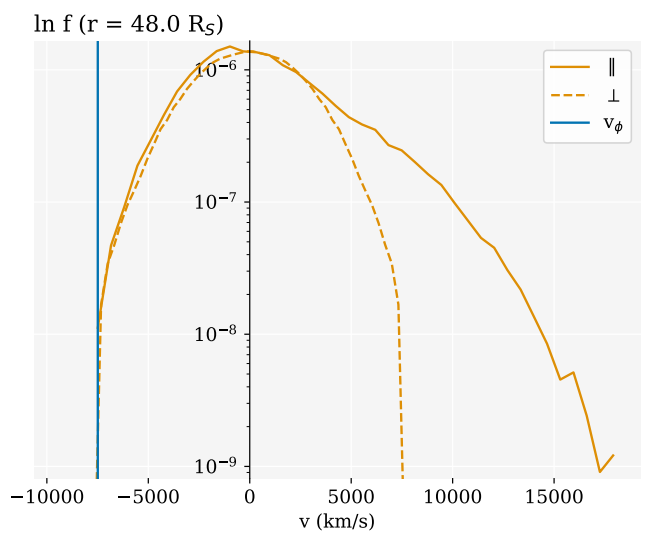

Figure 4. Parallel and perpendicular cuts through electron VDF, in the last radial bin of the simulation run MC, at a distance of $48 R_{S}$, plotted in the Sun's rest frame. The negative cutoff velocity is marked with a blue line.

advance up to a distance where their radial velocity becomes zero, and then start falling back towards the Sun, at every distance reaching the same absolute velocity as on the way up, only in the opposite direction. The velocity of electrons with the energy $e \phi$ :

$$
v_{\phi}(r)=\sqrt{\frac{2 e \phi(r)}{m_{e}}},
$$

thus represents a boundary in the sunward direction, the cutoff velocity below which no electrons are found. $v_{\phi}$ is defined in the Sun's rest frame.

The electric potential difference obtained in the simulation is not the total electric potential supposed to be present in the solar wind, but the potential difference between a given radial distance and the top simulation boundary $\left(\Delta \phi(r)=\phi_{\text {top }}-\phi(r)\right)$. To obtain the total electric potential, and not only the potential over the simulation length, we estimated the potential difference between the top boundary and infinity, or interstellar medium $\left(\phi_{\infty-t o p}\right)$. The ambipolar electric field is the strongest close to the Sun where the solar wind acceleration is the fastest, and decreases with radial distance with a power law between 1 and 2 . Therefore $\phi(r)$ asymptotically approaches zero for large radial distances and $\phi_{\infty-t o p}$ is relatively small.

Fist we estimated $\phi_{\infty-t o p}$ from the electron VDF in the last radial bin. We use the exospheric model prediction and look for the cutoff electron velocity in the sunward direction (see Fig. 4). Technically this cutoff velocity is determined by the electron VDF prescribed at the upper boundary $\left(T_{e, t o p}\right)$. Even though $T_{e, t o p}$ is an input parameter, it is dependant on the conditions set at the bottom boundary, and was found trough iteration towards a stationary solution conserving fluxes of both species. As $T_{e, t o p}$ is the same for runs $\mathrm{HC}, \mathrm{MC}$ and LC, so is the cutoff velocity in the last radial bin: $v_{\phi, t o p}=-7490 \mathrm{~km} / \mathrm{s}$. This velocity corresponds to electric potential $\phi_{\infty-t o p}=159 \mathrm{~V}$.

The estimation of $\phi_{\infty-t o p}$ can also be found from the radial extrapolation of $E$ measured in the simulation runs. To predict the behaviour of $E$ for the distances above the top boundary, existing values were fitted with a power law function:

$$
f_{E}(r)=a \cdot r^{b},
$$




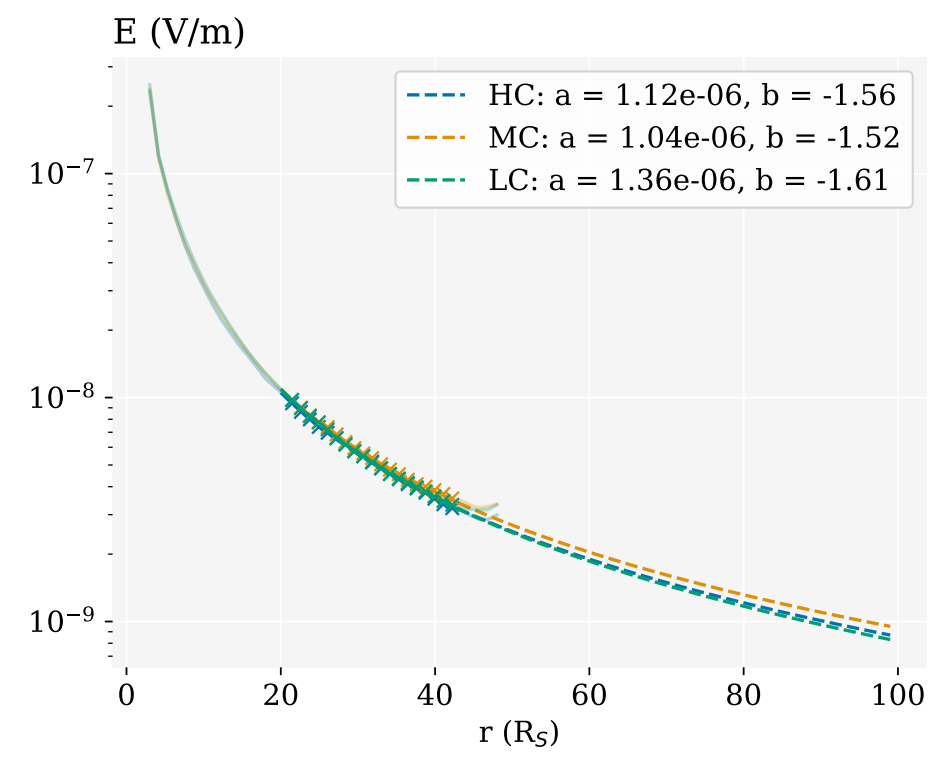

Figure 5. The extrapolation of $E$ above the top simulation boundary. $E$ measured in the simulation runs $\mathrm{HC}, \mathrm{MC}$ and LC is shown with a pale full line, crosses denote the points used for the fitting with Eq. 12, and the dashed lines the fitted curves. The obtained fitting parameters are shown in the legend.

where $a$ and $b$ are the fitting parameters. The fit was preformed only to the points above the distance of $21 R_{S}$ to avoid regions of strong solar wind acceleration. Acceleration contributes to the total value of $E$, and only above the acceleration region we expect for $E$ to evolve as a power law with the radial distance. An upper radial distance limit was set to $44 R_{S}$, to avoid the effects of the simulation upper boundary. The results of the fitting procedure are shown in Fig. 5, where the fitted values are marked by crosses and the dashed line represents the obtained fit for each of the three simulation runs. The obtained fitting parameters $(a$ and $b)$ are marked in the legend. $\phi_{t o p, \infty}$ is then obtained by integration of Eq. 12 on the interval between $49 R_{S}$ and $\infty$. The resulting $\phi_{t o p, \infty}$ are very close to the one estimated from electron VDF, amounting to 159,181 , and $144 \mathrm{~V}$, for simulation runs $\mathrm{HC}, \mathrm{MC}$, and $\mathrm{LC}$, respectively.

Even though $\phi_{t o p, \infty}$ is not a direct output of the simulation, we are confident in the obtained values, as the two different estimation approaches give very similar results. For simplicity, the value $\phi_{\infty-t o p}=159 \mathrm{~V}$ obtained from electron VDFs, is used in further analysis.

The absolute value of ambipolar electric field obtained by the simulation is compared to the Dreicer electric field $\left(E_{D}\right)$ (Dreicer, 1959), a measure of electric field strength required for an electron with a kinetic energy of $\frac{3}{2} k_{B} T_{e}$ to gain the energy of $k_{B} T_{e}$ in one mean-free-collision time. $E_{D}$ is defined as:

$$
E_{D}=\frac{k_{B} T_{e, \text { core }}}{e \lambda_{m f p}},
$$

where $\lambda_{m f p}$ stands for the mean-free path, which is calculated as the ratio of electron thermal velocity $\left(v_{e, t h}\right)$ and electron - proton collision frequency $\left(\nu_{e, p}\right)$ measured in the simulation. 

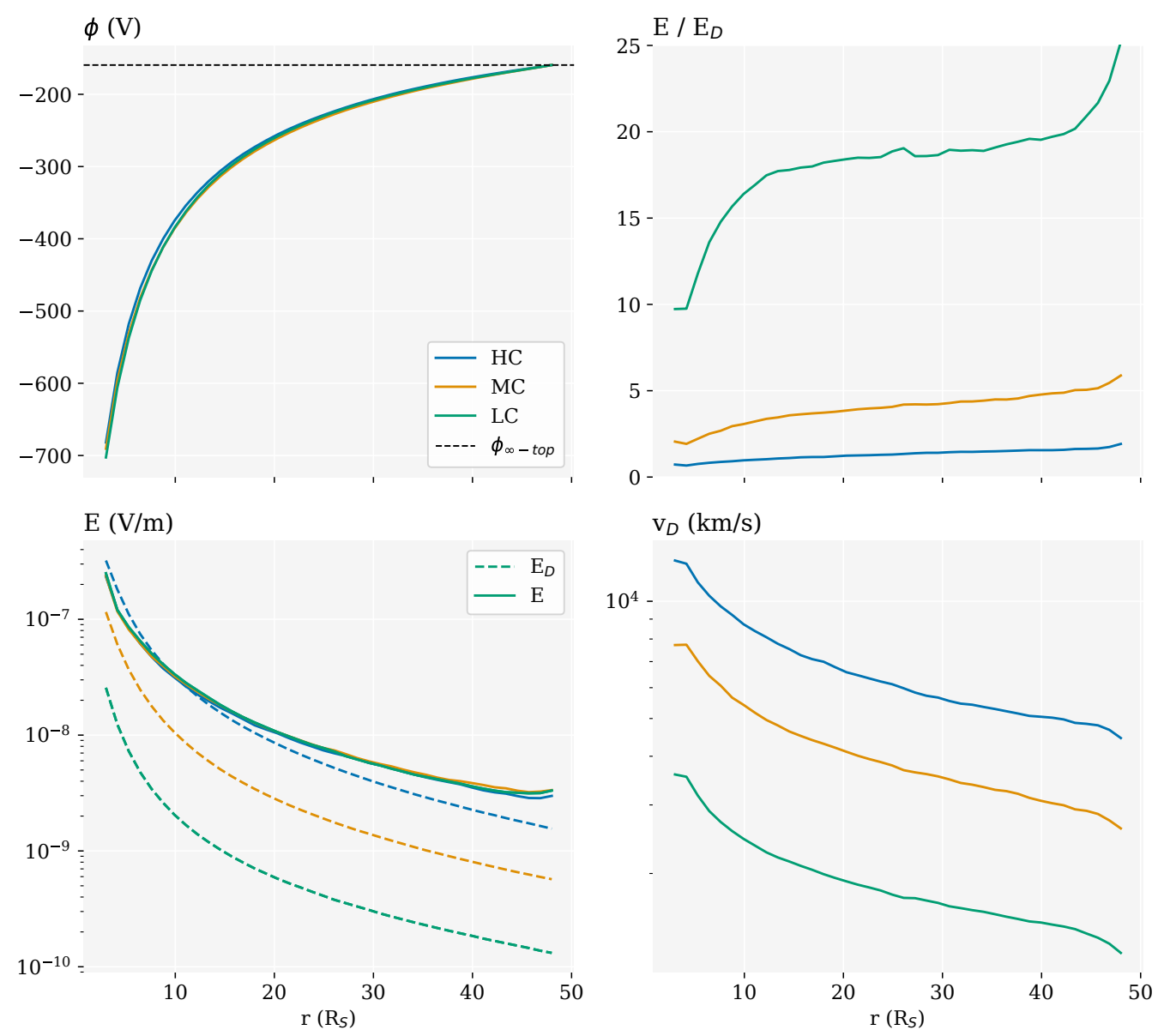

Figure 6. (a) Electric potential measured in the simulations and shifted for the estimated potential above the top simulation boundary $\left(\phi_{\infty-t o p}\right)$, (b) Ambipolar electric field (E) (full line) and Dreicer electric field (dashed line), (c) The ratio between ambipolar and Dreicer electric field, (d) separation velocity $\left(v_{D}\right)$.

Following the works of Fuchs et al. (1986); Scudder (1996), the electron velocity space can be separated into two regions by a boundary velocity defined as:

$$
v_{D}=\sqrt{\frac{3 k_{B} T_{e}}{m_{e}} \cdot \frac{2 E_{D}}{E}},
$$

where $\mathrm{E}$ is the total, ambipolar electric field. Electrons with velocity lower than $v_{D}$ defined in the ion rest frame, collide frequently enough for the electric force to be overdamped with Coulomb collisions, preserving a Maxwellian shaped VDF. Electrons with velocity higher than the defined boundary are underdamped by collisions and experience an acceleration by the electric force, becoming the so called, runaway electrons.

\subsection{Results}

The radial evolution of electric potential $(\phi)$ and electric field $(E)$ is shown in Fig. $6(\mathrm{a}, \mathrm{b})$. While both of these quantities remain very similar for the three simulations, 

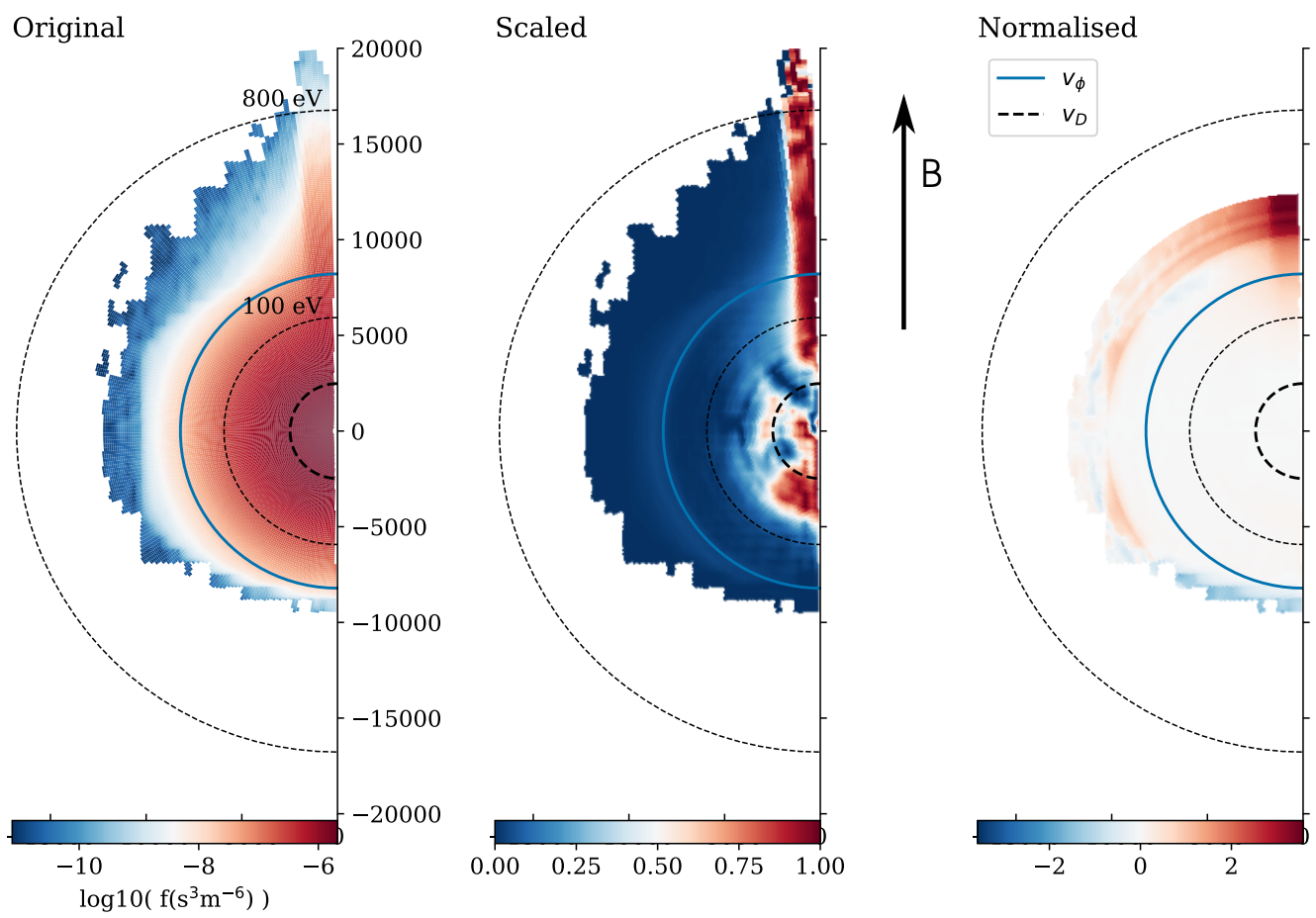

Figure 7. Two-dimensional representation of a gyrotropic electron VDF in the 28th radial bin (35 $R_{S}$ ) of the simulation run MC. The original electron VDF is shown on the left, a scaled VDF in the middle, and a normalised VDF on the right. We use the core electron resting frame where magnetic field is aligned with the y-axis. The electric potential velocity $\left(v_{\phi}\right)$ and the Dreicer velocity $\left(v_{D}\right)$ are marked with blue and black lines.

a strong variation is seen for the Dreicer electric filed $\left(E_{D}\right)$, a parameter comparing electric field with the collisionality of the system. Accordingly, the ratio $E / E_{D}$ reaches the highest values for the least collisional case ( $\sim 20$ in run LC), and stays on the order of 1 for the most collisional case (run HC, Fig. 6 (c)). Fig. 6 (d) shows the velocity $v_{D}$ defined in the previous section, separating the over- and underdamped regions of the VDF.

We compare the calculated separation velocities $v_{\phi}$ and $v_{D}$ with the measured electron VDFs. A new representation method introduced by Behar et al. (2020) is used to highlight higher order VDF features and their departures from isotropy. Left plot in Fig. 7 displays an original gyrotropic VDF from the simulation run MC. A 2-dimensional linear interpolation between the sampled points was used, resulting in a smoother and more continuous plot. Logarithmic colour scale allows a recognition of the typical electron VDF features: a dense and isotropic core component and a beam-like strahl at positive velocity values. The middle plot shows the same VDF in the scaled representation, where each energy bin - each circular belt in the $\left(v_{\|}, v_{\perp}\right)$ parameter space - is scaled to the values between 0 and 1 . With this representation we lose the information about the absolute value of $f$ and its strong gradient along the energy dimension, but we expose the smaller anisotropic features at all energies. In cases where two features arise in the same energy bin, the scaled VDFs can be misleading, only highlighting the bigger feature. The right plot shows the normalised representation, where the values are normalised to the perpendicular cut through electron $\operatorname{VDF}\left(f_{\perp}=f\left(v_{\|}=0\right)\right)$. Regions of VDF where the density flux is lower than 
(a) $\ln f\left(r=16.8 R_{S}\right)$

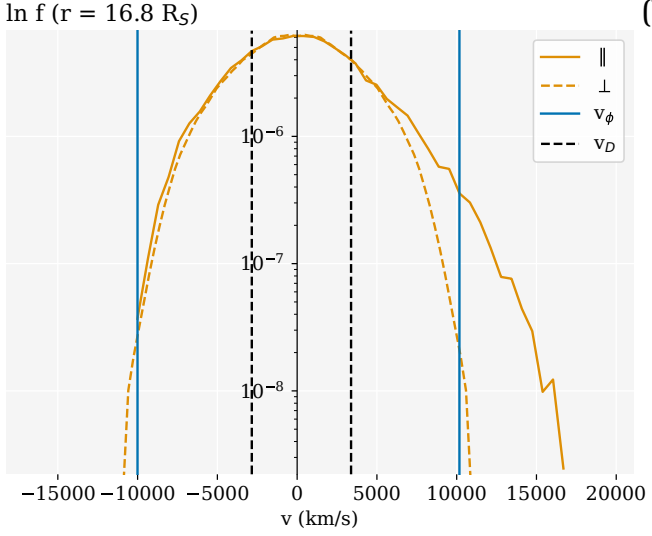

(c) $\ln f\left(r=35.3 R_{S}\right)$

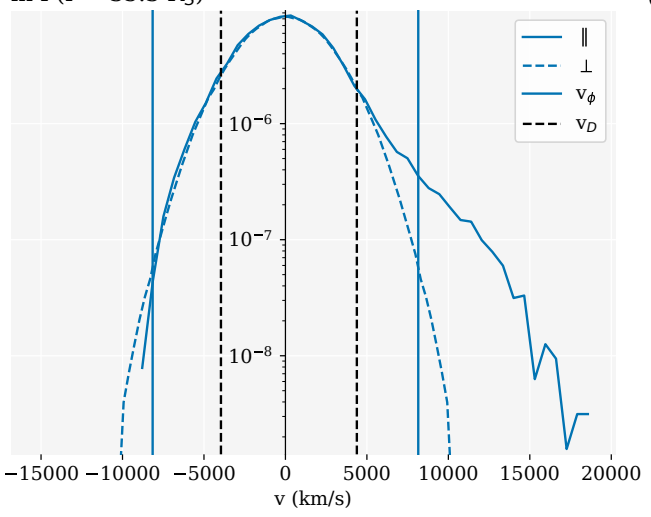

(b) $\ln f\left(r=35.3 R_{S}\right)$

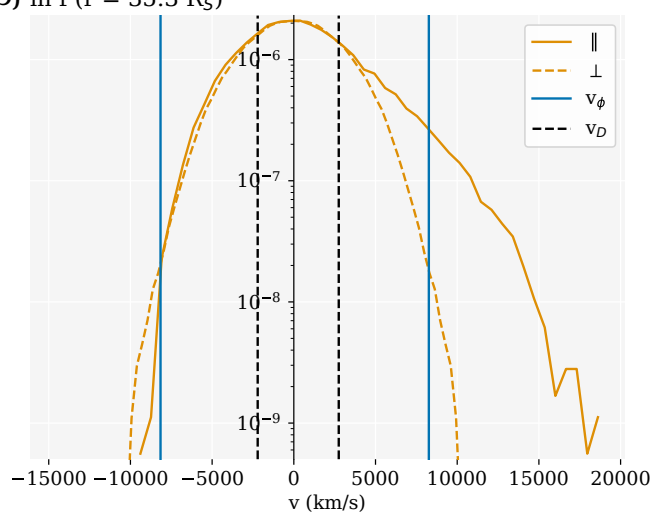

(d) $\ln f\left(r=35.3 R_{S}\right)$

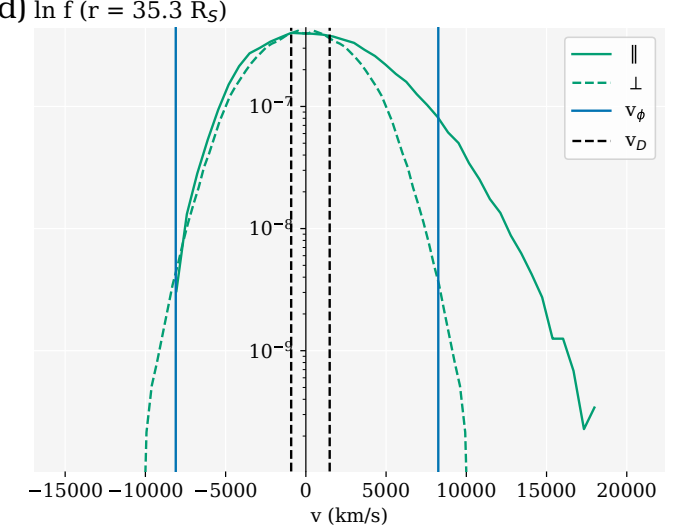

Figure 8. Parallel and perpendicular cuts through an electron VDF at the distance of $17 R_{S}$ (a) for the simulation run $\mathrm{MC}$, and at $35 R_{S}$ (right) for the simulation runs $\mathrm{HC}$ (c), MC (b), and LC (d). The cuts are plotted in core electron resting frame. $v_{\phi}$ and $v_{D}$ are indicated with blue and black lines. 
along the perpendicular direction appear in blue and regions with higher density in red. With this representation the small VDF features are less pronounced than in the scaled VDF, however a relation with the original VDF is preserved through a norm, in this case chosen to be $f_{\perp}$. VDFs are shown in electron core resting frame, as this is the frame in which isotropy is expected.

The scaled distribution reveals two features aligned with magnetic field: the strahl present at positive velocities, and another overdensity at small negative velocities. The second feature is very small and does not appear in the normalised representation. It results from a slight mismatch between the anti-sunward portion of electron VDF leaving the simulation at the top boundary and the sunward portion defined with input parameters.

$v_{D}$ and $v_{\phi}$ are overplotted as half circles with dashed black, and full blue line, respectively. Positive signed $v_{D}$ corresponds to the velocity where first strahl electrons are found (see the scaled representation), while negative signed $v_{\phi}$ coincides with the cutoff, clearly seen in blue in the normalised representation. Since electron core is close to isotropic and drifting with a relatively low speed, positive signed $v_{\phi}$ also corresponds to the upper velocity limit of the core population. The same conclusions follow from the electron VDF slices at two different radial distances shown in Fig. 8 (a, b).

We are interested in the behaviour of electron VDF parallel to the magnetic field, thus we average the values within a pitch-angle $10^{\circ}$ to create parallel cuts through the VDF in original, scaled and normalised representation. These values are then plotted with respect to the radial distance in Fig. 9, for the simulation run MC. This plotting technique allows us to observe the radial evolution of the core and the strahl component. Over all radial distances positive $v_{D}$ follows the transition between the core and the strahl component (see scaled representation), while negative $v_{\phi}$ follows the exospheric cutoff (see normalised representation). The same type figures for simulation runs $\mathrm{HC}$ and LC are added in Appendix A.

We compare the cuts through electron VDF at the same radial distance, in three different simulations in Fig. 8 (b, c, d). The first notable difference is the break-point velocity between the core and the strahl electrons. In the more collisional run $\mathrm{HC}$ the collisions are able to maintain a Maxwellian VDF up to higher velocity compared to the less collisional runs MC and LC. While $v_{\phi}$ is almost the same for all the runs, $v_{D}$ reflecting the collisionality of the system varies between the runs.

Both, positive and negative signed velocities $v_{\phi}$ and $v_{D}$, are marked on all plots because they are expected to describe the VDF in both senses. In the antisunward direction $v_{\phi}>v_{D}$ means that the electrons with energies smaller than the local potential energy, which will eventually be slowed down and start falling back towards the Sun, already exhibit non-Maxwellian features. Whether this results in a non-Maxwellian sunward directed portion of electron VDF can not be determined with the results obtained from our model. The sunward portion of the VDF is defined at the top boundary and is assumed to be Maxwellian.

\section{Pitch-angle width (PAW) \& strahl parallel temperature $\left(T_{s, \|}\right)$}

\subsection{Method}

We define the strahl as the residual anti-sunward component of the electron velocity distribution function and we characterise it with two parameters, the pitchangle width (PAW) and the strahl parallel temperature $\left(T_{\|}\right)$, in the same way as in the observational studies by Berčič et al. (2019); Berčič et al. (2020). PAW width is obtained as a full width half maximum (FWHM) of the pitch-angle distributions in an 

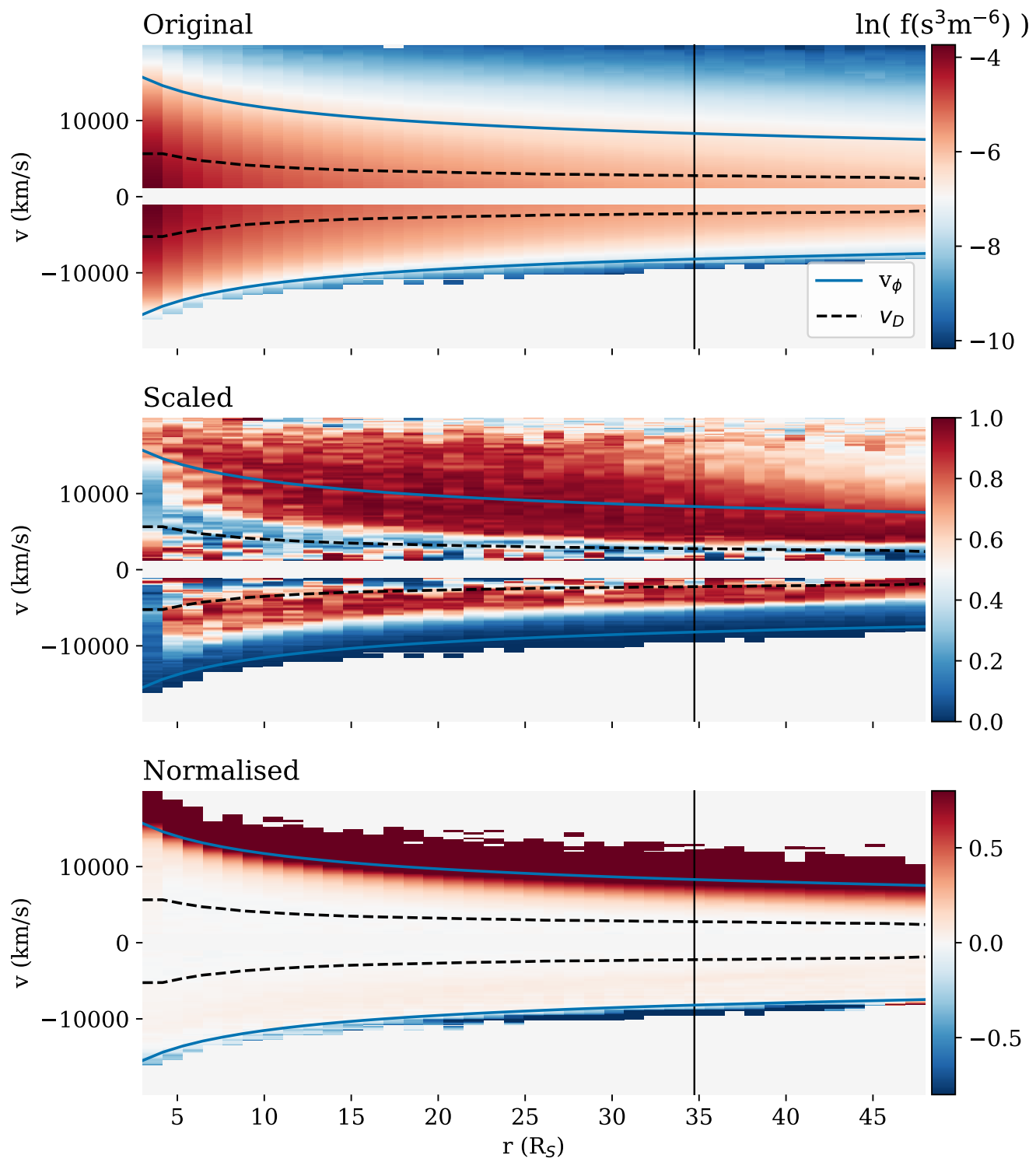

Figure 9. Parallel cuts through electron VDF plotted with respect to the radial distance in original (top), scaled (middle), and normalised (bottom) representation for the simulation run MC. $v_{\phi}$ and $v_{D}$ are marked with blue and black lines. A black vertical line denotes the radial distance of the VDFs shown in Figs. 7 and 8 (right). 

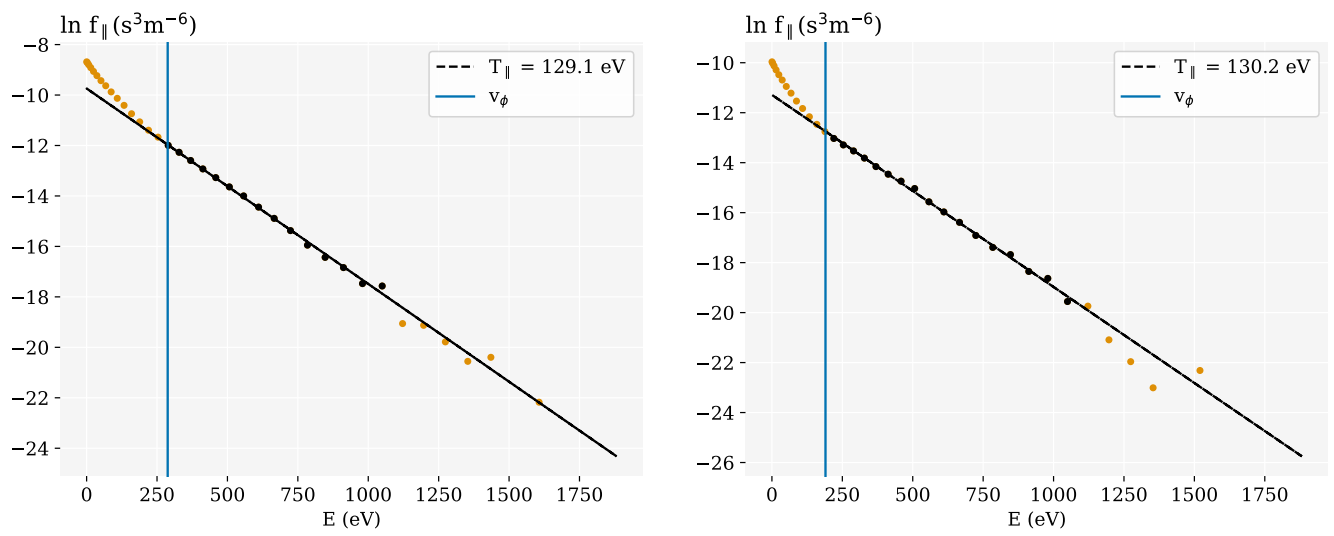

Figure 10. An example of the Maxwellian fit to the parallel strahl VDF $\left(f_{\|}\right)$to obtain $T_{s, \|}$, shown for simulation run MC at radial distances $17 R_{S}$ (left), and $35 R_{S}$ (right). The data points not included in the fit are marked with yellow and the data points included in the fit with black. The black dashed line shows the fit with the resulting $T_{s, \|}$ marked in the legend, and the blue line denotes the assumed separation velocity between the core and the strahl component.

energy bin:

$$
f_{i}(\alpha)=f_{\text {max }, i} \cdot \exp \left(-\frac{\alpha^{2}}{2 \sigma_{i}^{2}}\right), \quad P A W_{i}=2 \sqrt{2 \ln 2} \cdot \sigma_{i},
$$

where $\alpha$ is the pitch angle and index $i$ denotes different energy bins. We arbitrarily define 20 logarithmically spaced energy bins between energies 79 and $3162 \mathrm{eV}$. Logarithmic spacing was used to provide a better comparison between the simulation and observational data, as electrostatic analysers normally sample electron energies in this way.

$T_{s, \|}$ is obtained by fitting a 1-dimensional Maxwellian to the VDF integrated along the perpendicular direction $\left(f_{\|}=\int f\left(v_{\|}, v_{\perp}\right) d v_{\perp}\right)$ in the logarithmic space:

$$
\ln f_{\|}\left(v_{\|}\right)=-\frac{m_{e}}{2 k_{B} \cdot T_{s \|}} \cdot v_{\|}^{2}+\ln \left(n_{s} \sqrt{\frac{m_{e}}{2 \pi k_{B} \cdot T_{s \|}}}\right) .
$$

The fit is preformed only to the antisunward portion of electron velocity space dominated by the strahl electron population (see Fig. 10). We found that $v_{\phi}$ in the sunward and anti-sunward direction describes well the properties of the electron core. Therefore we use it as the separation velocity between the core dominated and strahl dominated portions of electron VDF. An upper energy limit for the energies included in the $T_{s, \|}$ fit was arbitrarily set to $1274 \mathrm{eV}$ to avoid inclusion of the noise.

\subsection{Results}

The comparison of PAWs at the radial distance of $35 R_{S}$ for the three simulation runs shown in Fig. 11 reveals that Coulomb collisions only affect the lower energy strahl electrons. The first plotted PAW value denotes the energy at which the PAW of the electron VDF drops below $180^{\circ}$, marking the boundary between the core and the strahl electrons. The strahl break point energies are different for the three runs, as already observed from VDF slices (Fig. 8). The PAWs also exhibit different shapes with respect to the electron energy: the transition between broad strahl at lower electron energies, and narrow strahl at high energies is smoother for the more collisional case 


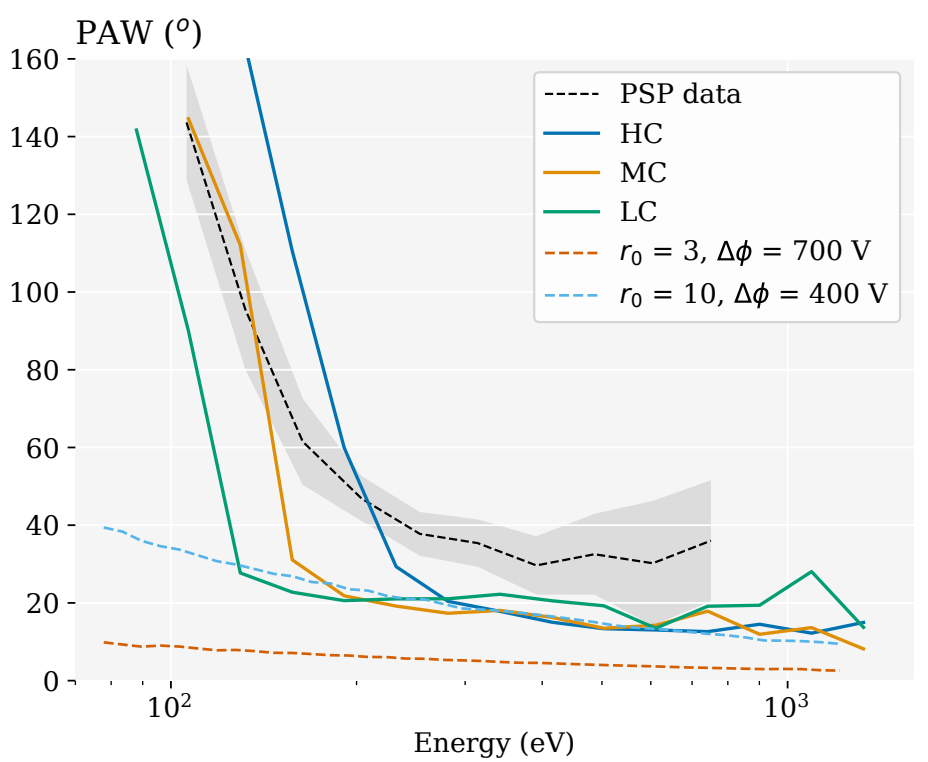

Figure 11. Strahl PAWs shown for electron VDFs at the radial distance of $35 R_{S}$ for the simulation runs $\mathrm{HC}, \mathrm{MC}$ and LC. The coloured dashed lines show PAWs obtained from collisionless single-exobase focusing model for different choices of the exobase $\left(r_{0}\right)$. Averaged PAW observed during the first two encounters of PSP in the low electron beta solar wind is shown with a black dashed line and a grey belt denoting the measurement error. The observational data was taken from Berčič et al. (2020).

$\mathrm{HC}$, and more abrupt for the less collisional cases MC, and LC. Above $\sim 250 \mathrm{eV}$ three PAW curves reach the same value, showing that collisionality of the system does not affect the high energy electrons.

Results of the collisionless single-exobase focusing model (see Eq. 6 in Berčič et al. (2019)) are also shown in Fig. 11 for two different sets of input parameters. The red dashed line shows the PAW obtained at $35 R_{S}$ if the exobase $\left(r_{0}\right)$ is set to $3 R_{S}$ and the potential difference $\Delta \phi=700 \mathrm{~V}$ (like in BiCoP runs). As it results on still much narrower strahl, we increased the exobase and decreased the potential difference accordingly. The result of a simple model that matches well PAWs obtained from all three simulation runs above $\sim 250 \mathrm{eV}$, and the least collisional run LC down to the energy $\sim 130 \mathrm{eV}$, was found for $r_{0}=10 R_{S}$, and $\Delta \phi=400 \mathrm{~V}$.

The black dashed line shows PAW values measured in the low electron beta solar wind $(<0.7)$ during the second encounter of PSP, shown in Berčič et al. (2020) - Fig. 5 (b). The observed strahls appear $10-20^{\circ}$ wider for the high electron energy part, but show a smooth transition between broad and narrow strahl, similar to the one found in the simulation run HC. The strahl break point found from PSP data appears at lower energy compared to the run $\mathrm{HC}$, but correlates well to the break point found for run MC.

An increase of $T_{s, \|}$ with radial distance was found in all three simulation runs. Fig. 12 shows electron VDFs integrated along the perpendicular direction $\left(f_{\|}\right)$at different radial distances normalised with a integrated Maxwellian VDF defined at the 


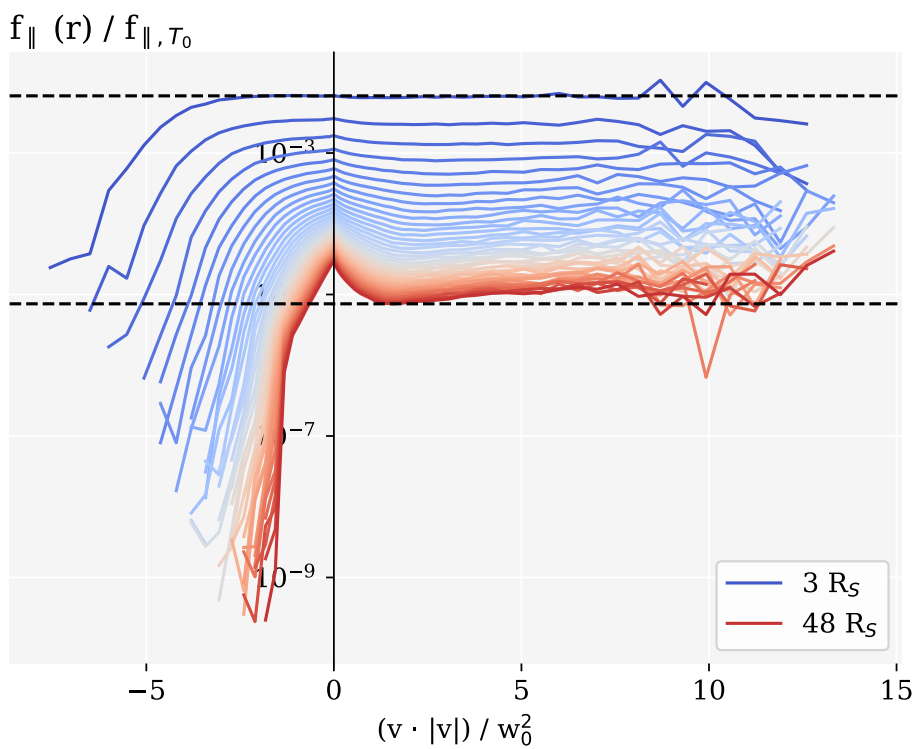

Figure 12. Electron VDFs, integrated along the $\perp$ direction $\left(f_{\|}\right)$, for different radial bins, normalised with a Maxwellian VDF with the temperature $T_{e, b o t}$. X-axis represents velocity $(v)$ multiplied with its absolute value in the units of square of thermal velocity of the electron VDF at the bottom boundary $\left(w_{0}^{2}\right)$. Radial distance is presented in colour spanning from blue closer to the Sun to red at the top boundary. Presented data is from the run MC, the same figures from runs $\mathrm{HC}$ and $\mathrm{LC}$ can be found in Appendix B.

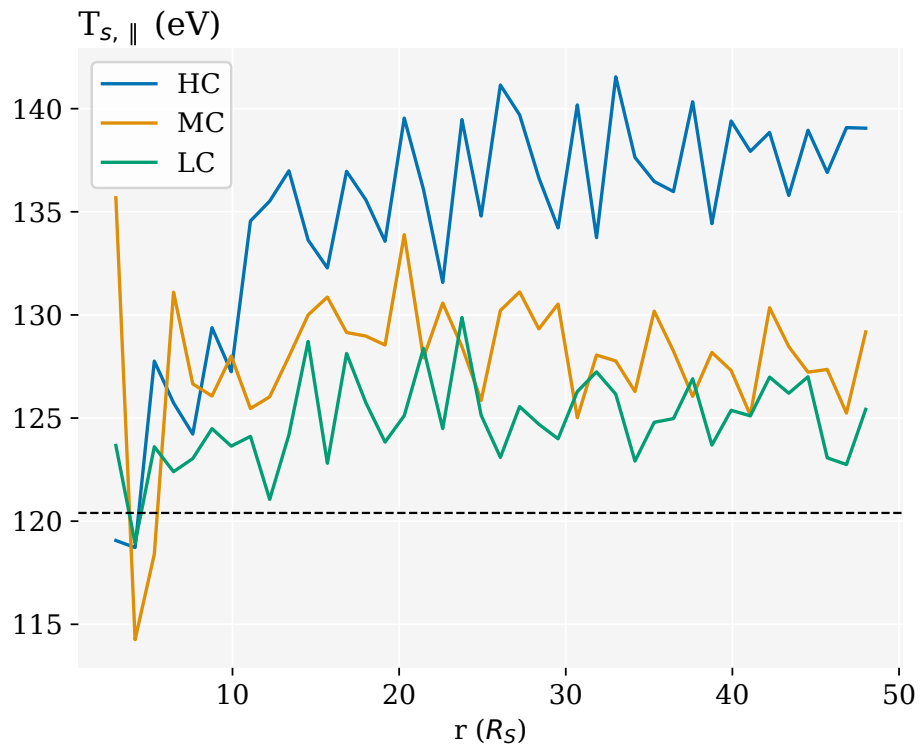

Figure 13. Evolution of $T_{\|}$over radial distance for the simulation runs HC, MC, and LC. The dashed black line shows the temperature of the Maxwellian set at the bottom boundary. 
bottom boundary $\left(f_{0, \text { Maxw }}\right)$ :

$$
l=\frac{\int f_{i}\left(v_{\|}, v_{\perp}\right) d v_{\perp}}{\int f_{0, M a x w}\left(v_{\|}, v_{\perp}\right) d v_{\perp}}
$$

where index $i$ is the number of the radial bin. This technique was used to verify the exospheric prediction, which says that $f_{\|}$should, in absence of collisions and wave-particle interactions, remain unchanged in the exosphere, and carry the information about the shape of the VDF at the exobase to farther radial distances. If $T_{s, \|}$ remains unchanged from the bottom boundary the presented normalisation results in a horizontal line, as found for the VDF in the first radial bin (blue colour). Decreasing curves denote temperatures smaller than $T_{e, b o t}$, which can be seen for farther radial distances (red colour) at low electron energies and represent the electron core population. Increasing curves appearing at strahl electron energies indicate that the $T_{s, \|}$ slightly increases with radial distances. Fig. 12 includes values from the run $\mathrm{MC}$, while plots for runs $\mathrm{HC}$ and $\mathrm{LC}$ are added in Appendix B.

The same result was obtained by fitting $f_{\|}$with a $1 \mathrm{D}$ Maxwellian to obtain $T_{s, \|}$ (see Fig. 13). The increase in $T_{s, \|}$ is the largest for the most collisional run $\mathrm{A}$, at radial distance of $35 R_{S}$ by $15 \%$ exceeding the initial $T_{e, b o t}$. The smallest increase was found in run $\mathrm{C}$, amounting to $3 \%$.

\section{Discussion}

\subsection{Modelled and observed solar wind}

The used kinetic solar wind model does not capture all the physics of the solar wind. Most importantly it does not account for electro-magnetic (EM) wave activity, or the Parker spiral, non-radial, magnetic field. It assumes spherically geometric radial expansion to reconstruct a 3-dimensions in space from its 1-dimensional simulation domain. However, it allows us to focus on electron kinetic physics on the global solar wind scales. Using this model we are able to quantify the contribution of the kinetic electron behaviour, under the influence of gravity and Coulomb collisions, in the solar wind dynamics. As the resulting electron VDFs are not far from the observed ones, we can speculate that the recognised differences between the modelled and observed VDF are the result of the physical mechanisms not included in our simulation, like EM waves or non-radial magnetic field.

The simulation run A presents the solar wind arising solely from the hot Maxwellian solar corona with a temperature of $2 \mathrm{MK}(172 \mathrm{eV})$. This temperature is higher than value 0.79 MK reported above the surface for the coronal holes (David et al., 1998; Cranmer, 2002), but an upper limit temperature related to the edges of coronal holes in the recent study by Berčič et al. (2020) inferring the temperature of the coronal electrons from the strahl electrons measured by PSP. The estimated density at $1 R_{S}$ in the simulation is about one order of magnitude lower than that reported for the coronal holes, measured by multi-frequency radio imaging (Mercier \& Chambe, 2015). Due to their small mass, the contribution of electrons to the total mass flux of the solar wind is very small, however, the high velocities they reach, and their subsonic behaviour have an important role in the solar wind acceleration. In comparison to the heavier protons, electrons evaporate from the Sun faster, which requires an existence of large-scale electric field ensuring the plasma quasi-neutrality (Lemaire \& Scherer, 1971). This electric field is referred to as the ambipolar electric field (E), and is self-consistently obtained in the simulation. It is responsible for acceleration of the solar wind protons to the supersonic velocity at $4 R_{S}$, and to the terminal velocity of $206 \mathrm{~km} / \mathrm{s}$. Even though the modelled corona is somewhat hotter than measured, the obtained terminal velocity is still about a third smaller than frequently observed 



(a)

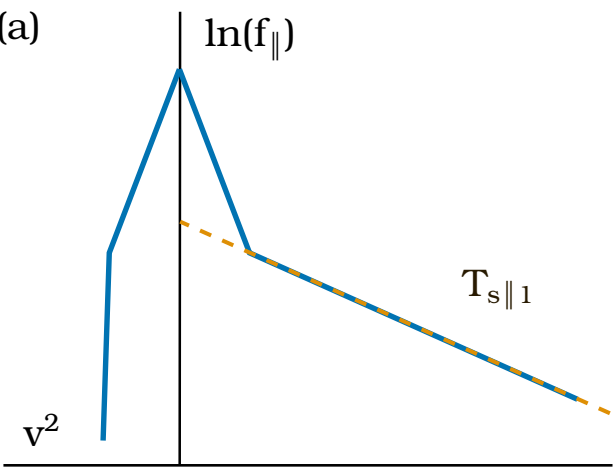

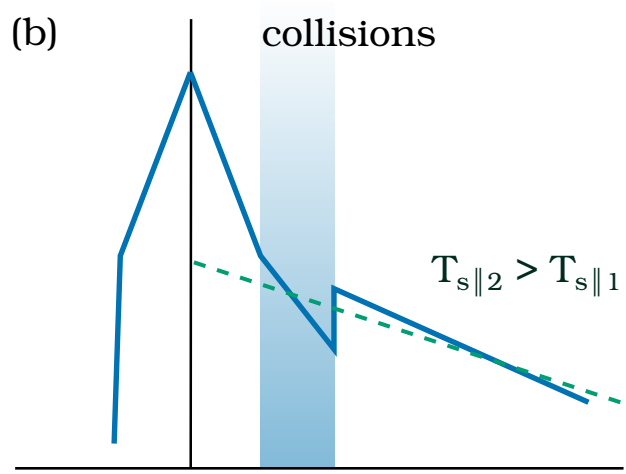

Figure 14. An illustration of how Coulomb collisions can increase $T_{s, \|} \cdot$ (a) Collisionless case, (b) collisions decrease the temperature of only lowest energy strahl electrons, which results in the increase of the total effective $T_{s, \|}$.

With a schematics in Fig. 14, we propose a physical mechanism which could result in an increase of $T_{s, \|}$ with radial distance. The parallel cut through electron VDF is illustrated with straight lines in the logarithmic parameter space, representing Maxwellians with different temperatures. Fig. 14 (a) shows the core and the strahl for a collisionless case, where a yellow dashed line represents the fit giving $T_{s, \|}$. The same VDF cut is shown in Fig. 14 (b) for a collisional case, where the lowest strahl energies are affected by Coulomb collisions. In the region marked with blue, the strahl electrons are cooled down by collisions, however, when fitting to the whole strahl energy range (green dashed line), the obtained temperature is higher than the one obtained for the collisionless case (a). In the simulation this mechanism, exaggerated in the schematics, is continuous, reshaping the the parallel cut through the strahl VDF over the radial distance. The strahl parallel profiles obtained by the kinetic model are well represented by a Maxwellian, however, it is not obvious why a mechanism described above would preserve a Maxwellian shape.

Comparing the simulation results with the observations shown by Berčic et al. (2020), we believe that most of the solar wind observed during the first two encounters of PSP best corresponds to the simulation runs HC or MC. Therefore the presented $T_{s, \|}$ (Berčič et al. (2020) - Figs. 6 and 7) probably overestimates the temperature of coronal electrons. In the simulation runs $\mathrm{HC}$ and $\mathrm{MC}$ at the distance of $\sim 35 R_{S}$, $T_{s, \|}$ is overestimated by $15 \%$ and $8 \%$, respectively. Applying this correction to the observed $T_{s, \|}$ with a mean value of $96 \mathrm{eV}$, we obtain the mean temperature of coronal electrons between 83 an $89 \mathrm{eV}$.

\section{Conclusions}

We presented results of a kinetic model of the solar wind accounting for binary Coulomb collisions $(\mathrm{BiCoP})$, simulating the solar wind acceleration region $\left(1-45 R_{S}\right)$. The model does not include EM waves and non-radial magnetic fields. Nevertheless it can produce a solar wind, accelerated only through the ambipolar electric field $(E)$, rising from the difference in the pressure gradients between electrons and protons. High coronal temperatures were assumed, leading to the terminal solar wind velocities approximately a third smaller than the ones reported by PSP. We conclude that, while $E$ is responsible for a big part of solar wind terminal velocity, it is not the only solar wind acceleration mechanism. 
The self-consistently obtained $E$ in our model was found to be on the order of the Dreicer electric field $\left(E_{D}\right)$. We analysed the effects it has on electron VDF. The cumulative effects of $E$ were predicted by exospheric solar wind models, and the separation velocity $v_{\phi}$ correlates well with the electron sunward cutoff velocity. Similarly, $v_{\phi}$ describes an upper velocity limit for the core population in the antisunward direction. The local effects of $E$ on the VDF were described by the Steady Electron Runaway Model (SERM) (Scudder, 2019b) predicting a separation of electron velocity space into two regions separated by $v_{D}$ : an overdamped region, where collisions are frequent enough to overdamp the electric force and preserve a Maxwellian VDF, and an underdamped region, where electrons can be accelerated by $\mathrm{E}$ and departures from a Maxwellian VDF can be found. In our obtained VDFs $v_{D}$ represents well the strahl break point velocity.

Strahl focusing in the kinetic model is compared to the simple, single-exobase collisionless focusing model. We find that at the distance of $34 R_{S}$, energies above $250 \mathrm{eV}$ are not affected by Coulomb collisions. Pitch-angle widths are observed to be larger than the ones obtained from a simple focusing model, and this difference is accounted to the multi-exobase phenomena. For energies below $250 \mathrm{eV}$ Coulomb collisions are able to scatter the strahl electrons and change the dependence of PAW on electron energy.

In the collisionless approximation the strahl parallel temperature $\left(T_{s, \|}\right)$ is independent of radial distance. However, $T_{s, \|}$ in our simulation runs was found to be larger than the temperature set at the bottom boundary, and the increase to be correlated to the collisionality of the system. We presented a raw idea of how scattering of the low energy strahl electrons by Coulomb collisions in the solar wind acceleration region could affect $T_{s, \|}$.

\section{Appendix A Radial evolution of the parallel cuts through electron VDF for simulation runs $\mathrm{HC}$ and $\mathrm{LC}$}

\section{Appendix B $f_{\|}$normalised to the Maxwellian at the bottom bound- ary for simulation runs $\mathrm{HC}$ and $\mathrm{LC}$}

\section{Appendix C Simulation run with a non-Maxwellian top boundary con- dition}

With slices through electron VDFs at different radial distances we demonstrate the propagation of the non-Maxwellian feature produced in the sunward portion of the electron VDF at the top boundary. The parameters used for the presented run are gathered in Table C1. In this simulation run, $v_{D}$ (black dashed line in Fig. C1) separates the over-, and underdamped parts of the VDF in both directions. In the antisunward direction it marks the beginning of the strahl component, as already shown for runs $\mathrm{HC}, \mathrm{MC}$, and LC. In the sunward direction $v_{D}$ follows the beginning of the feature propagating towards the Sun, separating electron VDF into Maxwellian and non-Maxwellian parts.

\section{Acknowledgments}

The simulation data used in this work is publicly available: HC run (https://doi .org/10.6084/m9.figshare.13160114.v1), MC run (https://doi.org/10.6084/ m9.figshare.13160132.v1), and LC run (https://doi.org/10.6084/m9.figshare $.13160102 . v 1)$. This work was supported by the Programme Nationale Soleil Terre of Centre National de la Recherche Scientifique (CNRS/INSU). All the analysis was done, and the plots produced using open source Python libraries Numpy, Matplotlib, and Scipy. 
Table C1. Presented simulation runs and their crucial input parameters.

\begin{tabular}{cc|c}
\hline Parameters & Unit & non-Maxw. \\
\hline $\mathrm{N}$ & & 22500 \\
$\mathrm{v}_{C}$ & $v_{t h, 0}$ & 0.3 \\
$\mathrm{~T}_{e, p, b o t}$ & $10^{6} \mathrm{~K}$ & 1 \\
$\mathrm{~T}_{e, t o p}$ & $10^{6} \mathrm{~K}$ & 0.4 \\
$\mathrm{~g}_{0}$ & & 0.0177 \\
$\mathrm{r}$ & $\mathrm{R}_{S}$ & $4-49$ \\
$\mathrm{v}_{b o t}$ & $\mathrm{~km} / \mathrm{s}$ & 77 \\
$\mathrm{v}_{\text {top }}$ & $\mathrm{km} / \mathrm{s}$ & 171 \\
\hline
\end{tabular}

\section{References}

Behar, E., Sahraoui, F., \& Berčič, L. (2020). Resonant whistler-electron interactions: Mms observations versus test-particle simulation. Journal of Geophysical Research: Space Physics, 125(10), e2020JA028040. Retrieved from https:// agupubs .onlinelibrary.wiley.com/doi/abs/10.1029/2020JA028040 (e2020JA028040 10.1029/2020JA028040) doi: 10.1029/2020JA028040

Berčič, L., Maksimović, M., Landi, S., \& Matteini, L. (2019). Scattering of strahl electrons in the solar wind between 0.3 and 1 au: Helios observations. Monthly Notices of the Royal Astronomical Society, 486(3), 3404-3414. $\quad$ doi: 10.1093/ mnras/stz1007

Berčič, L., Larson, D., Whittlesey, P., Maksimović, M., Badman, S. T., Landi, S., ... Stevens, M. L. (2020, April). Coronal Electron Temperature Inferred from the Strahl Electrons in the Inner Heliosphere: Parker Solar Probe and Helios Observations. The Astrophysical Journal, 892(2), 88. doi: $10.3847 / 1538-4357 / \mathrm{ab} 7 \mathrm{~b} 7 \mathrm{a}$

Brasseur, G., \& Lemaire, J. (1977, February). Fitting of hydrodynamic and kinetic solar wind models. Planetary and Space Science, 25(2), 201-203. doi: 10.1016/ 0032-0633(77)90028-9

Chamberlain, J. W. (1960, January). Interplanetary Gas.II. Expansion of a Model Solar Corona. The Astrophysical Journal, 131, 47. doi: 10.1086/146805

Cranmer, S. (2002). Coronal holes and the solar wind. COSPAR Colloquia Series(January), 1-10. Retrieved from http://www.sciencedirect.com/ science/article/pii/S0964274902800038 doi: 10.1016/S0964-2749(02) 80003-8

David, C., Gabriel, A. H., Bely-Dubau, F., Fludra, A., Lemaire, P., \& Wilhelm, K. (1998). Measurement of the electron temperature gradient in a solar coronal hole. Astronomy and Astrophysics, 336(3), 90-94.

Dreicer, H. (1959, July). Electron and Ion Runaway in a Fully Ionized Gas. I. Physical Review, 115(2), 238-249. doi: 10.1103/PhysRev.115.238

Dreicer, H. (1960, January). Electron and Ion Runaway in a Fully Ionized Gas. II. Physical Review, 117(2), 329-342. doi: 10.1103/PhysRev.117.329

Feldman, W. C., Asbridge, J. R., Bame, S. J., Montgomery, M. D., \& Gary, S. P. (1975). Solar wind electrons. Journal of Geophysical Research. doi: 10.1029/JA080i031p04181

Fox, N. J., Velli, M. C., Bale, S. D., Decker, R., Driesman, A., Howard, R. A., ... Szabo, A. (2016). The Solar Probe Plus Mission: Humanity's First Visit to Our Star. Space Science Reviews, 204(1-4), 7-48. Retrieved from http:// $\mathrm{dx}$.doi.org/10.1007/s11214-015-0211-6 doi: 10.1007/s11214-015-0211-6

Fuchs, V., Cairns, R. A., Lashmore-Davies, C. N., \& Shoucri, M. M. (1986, September). Velocity-space structure of runaway electrons. Physics of Fluids, 29(9), 
2931-2936. doi: 10.1063/1.865493

Graham, G. A., Rae, I. J., Owen, C. J., Walsh, A. P., Arridge, C. S., Gilbert, L., ... Waite, J. H. (2017). The evolution of solar wind strahl with heliospheric distance. Journal of Geophysical Research: Space Physics, 122(4), 3858-3874. doi: 10.1002/2016JA023656

Halekas, J. S., Whittlesey, P., Larson, D. E., Mcginnis, D., Maksimovic, M., Berthomier, M., ... Malaspina, D. M. (2019). Electrons in the Young Solar Wind: First Results from Parker Solar Probe. Astrophysical Journal.

Hammond, C. M., Feldman, W. C., McComas, D. J., Phillips, J. L., \& Forsyth, R. J. (1996). Variation of electron-strahl width in the high-speed solar wind: ULYSSES observations. Astronomy and Astrophysics, 316, 350-354. Retrieved from http://ukads.nottingham.ac.uk/abs/1996A\{\\&\}A...316..350H

Hefti, S., Zurbuchen, T. H., Fisk, L. A., Gloeckler, G., Larson, D., \& Lin, R. P. (1999). The transition from slow to fast solar wind: Charge state composition and electron observations. AIP Conference Proceedings, 471, $495 . \quad$ doi: $10.1063 / 1.58682$

Horaites, K., Boldyrev, S., \& Medvedev, M. V. (2019). Electron strahl and halo formation in the solar wind. Monthly Notices of the Royal Astronomical Society, 484 (2), 2474-2481. doi: 10.1093/mnras/sty3504

Horaites, K., Boldyrev, S., Wilson, L. B., Viñas, A. F., \& Merka, J. (2018). Kinetic theory and fast wind observations of the electron strahl. Monthly Notices of the Royal Astronomical Society, 474(1), 115-127. doi: 10.1093/MNRAS/ STX2555

Jagarlamudi, V. K., Alexandrova, O., Berčič, L., de Wit, T. D., Krasnoselskikh, V., Maksimovic, M., \& Štverák, Š. (2020, July). Whistler Waves and Electron Properties in the Inner Heliosphere: Helios Observations. The Astrophysical Journal, 897(2), 118. doi: 10.3847/1538-4357/ab94a1

Jockers, K. (1970). Solar Wind Models Based on Exospheric Theory. \aap, 6, 219. Retrieved from https://ui.adsabs.harvard.edu/abs/1970A\{\\&\}A.... $.6 .219 \mathrm{~J}$

Kajdič, P., Alexandrova, O., Maksimovic, M., Lacombe, C., \& Fazakerley, A. N. (2016). Suprathermal Electron Strahl Widths in the Presence of NarrowBand Whistler Waves in the Solar Wind. The Astrophysical Journal, 833(2), $172 . \quad$ Retrieved from http://stacks.iop.org/0004-637X/833/ $i=2 / a=172$ ?key=crossref .6e0cb686c031c19378090937228000f 2 doi: $10.3847 / 1538-4357 / 833 / 2 / 172$

Kasper, J. C., Bale, S. D., Belcher, J. W., Berthomier, M., Case, A. W., Chandran, B. D. G., ... Schwadron, N. A. (2019, December). Alfvénic velocity spikes and rotational flows in the near-Sun solar wind. Nature, 576(7786), 228-231. doi: 10.1038/s41586-019-1813-z

Kohl, J. L., Noci, G., Antonucci, E., Tondello, G., Huber, M. C. E., Cranmer, S. R., ... Suleiman, R. M. (1998, July). UVCS/SOHO Empirical Determinations of Anisotropic Velocity Distributions in the Solar Corona. The Astrophysical Journal Letters, 501(1), L127-L131. doi: 10.1086/311434

Lamy, H., Pierrard, V., Maksimovic, M., \& Lemaire, J. F. (2003, Jan). A kinetic exospheric model of the solar wind with a nonmonotonic potential energy for the protons. Journal of Geophysical Research (Space Physics), 108(A1), 1047. doi: $10.1029 / 2002 J A 009487$

Landi, S., Matteini, L., \& Pantellini, F. (2012). On the competition between radial expansion and coulomb collisions in shaping the electron velocity distribution function: Kinetic simulations. Astrophysical Journal, $760(2)$. doi: 10.1088/0004-637X/760/2/143

Landi, S., Matteini, L., \& Pantellini, F. (2014). Electron heat flux in the solar wind: Are we observing the collisional limit in the $1 \mathrm{AU}$ data? Astrophysical Journal Letters, 790(1), 1-5. doi: 10.1088/2041-8205/790/1/L12 
Landi, S., \& Pantellini, F. (2001). On the temperature profile and heat flux in the solar corona: Kinetic simulations. Astronomy \& Astrophysics, 372, 686-701. doi: 10.1051/0004-6361:20010552

Landi, S., \& Pantellini, F. (2003). Kinetic simulations of the solar wind from the subsonic to the supersonic regime. Astronomy and Astrophysics, 400, 769-778. doi: 10.1051/0004-6361:20021822

Landi, S., Pantellini, F., \& Matteini, L. (2010). Radial evolution of the electron velocity distribution in the heliosphere: Role of collisions. AIP Conference Proceedings, 1216(1), 218-222. Retrieved from https://aip.scitation.org/ doi/abs/10.1063/1.3395841 doi: 10.1063/1.3395841

Lemaire, J., \& Scherer, M. (1970). Model of the polar ion-exosphere. Planetary and Space Science, 18(1), 103 - $120 . \quad$ Retrieved from http:// www.sciencedirect.com/science/article/pii/003206337090070X doi: https://doi.org/10.1016/0032-0633(70)90070-X

Lemaire, J., \& Scherer, M. (1971). Kinetic models of the solar wind. Journal of Geophysical Research, 76, 7479-. doi: 10.1029/JA076i031p07479

MacNeil, A. R., Owen, C. J., \& Wicks, R. T. (2017). Tests for coronal electron temperature signatures in suprathermal electron populations at $1 \mathrm{AU}$. Annales Geophysicae, 35(6), 1275-1291. doi: 10.5194/angeo-35-1275-2017

Macneil, A. R., Owens, M. J., Lockwood, M., Štverák, Š., \& Owen, C. J. $\quad$ (2020, Jan). Radial Evolution of Sunward Strahl Electrons in the Inner Heliosphere. Solar Physics, 295(2), 16. doi: 10.1007/s11207-019-1579-3

Maksimovic, M., Pierrard, V., \& Lemaire, J. F. (1997, August). A kinetic model of the solar wind with Kappa distribution functions in the corona. Astronomy and Astrophysics, 324, 725-734.

Maksimovic, M., Zouganelis, I., Chaufray, J. Y., Issautier, K., Scime, E. E., Littleton, J. E., ... Elliott, H. (2005). Radial evolution of the electron distribution functions in the fast solar wind between 0.3 and 1.5 AU. Journal of Geophysical Research: Space Physics, 110(A9), 1-9. doi: 10.1029/2005JA011119

Mercier, C., \& Chambe, G. (2015, November). Electron density and temperature in the solar corona from multifrequency radio imaging. Astronomy and Astrophysics, 583, A101. doi: 10.1051/0004-6361/201425540

Pagel, C., Gary, S. P., de Koning, C. A., Skoug, R. M., \& Steinberg, J. T.

(2007). Scattering of suprathermal electrons in the solar wind: ACE observations. Journal of Geophysical Research, 112(A4), 1-11. Retrieved from http://www.agu.org/pubs/crossref/2007/2006JA011967.shtml doi: 10.1029/2006JA011967

Pannekoek, A. (1922, July). Ionization in stellar atmospheres (Errata: 2 24). Bulletin Astronomical Institute of the Netherlands, 1, 107.

Parker, E. N. (1958, November). Dynamics of the Interplanetary Gas and Magnetic Fields. The Astrophysical Journal, 128, 664. doi: 10.1086/146579

Pierrard, V., Maksimovic, M., \& Lemaire, J. (1999, August). Electron velocity distribution functions from the solar wind to the corona. Journal of Geophysical Research, 104(A8), 17021-17032. doi: 10.1029/1999JA900169

Pilipp, W. G., Miggenrieder, H., Montgomery, M. D., Mühlhäuser, K. H., Rosenbauer, H., \& Schwenn, R. (1987). Characteristics of electron velocity distribution functions in the solar wind derived from the Helios Plasma Experiment. Journal of Geophysical Research, 92(A2), 1075. Retrieved from http:// doi.wiley.com/10.1029/JA092iA02p01075 doi: 10.1029/JA092iA02p01075

Rosseland, S. (1924, June). Electrical state of a star. Monthly Notices of the Royal Astronomical Society, 84, 720-728. doi: 10.1093/mnras/84.9.720

Saito, S., \& Gary, P. S. (2007). All whistlers are not created equally: Scattering of strahl electrons in the solar wind via particle-in-cell simulations. Geophysical Research Letters, 34(1), 1-5. doi: 10.1029/2006GL028173

Salem, C., Hubert, D., Lacombe, C., Bale, S. D., Mangeney, A., Larson, D. E., \& 
Lin, R. P. (2003, March). Electron Properties and Coulomb Collisions in the Solar Wind at $1 \mathrm{AU}$ : Wind Observations. The Astrophysical Journal, 585(2), 1147-1157. doi: $10.1086 / 346185$

Scudder, J. D. (1996, June). Dreicer order ambipolar electric fields at Parker's steady state solar wind sonic critical point. Journal of Geophysical Research, 101(A6), 13461-13472. doi: 10.1029/96JA00189

Scudder, J. D. (2019a, November). The Long-standing Closure Crisis in Coronal Plasmas. The Astrophysical Journal, 885(2), 148. doi: 10.3847/1538-4357/ $\mathrm{ab} 48 \mathrm{e} 0$

Scudder, J. D. (2019b, November). Steady Electron Runaway Model SERM: Astrophysical Alternative for the Maxwellian Assumption. The Astrophysical Journal, 885 (2), 138. doi: 10.3847/1538-4357/ab4882

Scudder, J. D. (2019c, September). The Thermal Force in Astrophysical Plasmas: Current Free Coulomb Friction. The Astrophysical Journal, 882(2), 146. doi: $10.3847 / 1538-4357 / \mathrm{ab} 3348$

Štverák, Š., Maksimovic, M., Trávníček, P. M., Marsch, E., Fazakerley, A. N., \& Scime, E. E. (2009). Radial evolution of nonthermal electron populations in the low-latitude solar wind: Helios, Cluster, and Ulysses Observations. Journal of Geophysical Research: Space Physics, 114(5), 1-15. doi: 10.1029/2008JA013883

Tao, J., Wang, L., Zong, Q., Li, G., Salem, C. S., Wimmer-Schweingruber, R. F., ... Bale, S. D. (2016, Mar). Quiet-time Suprathermal $(\sim 0.1-1.5 \mathrm{keV})$ Electrons in the Solar Wind. The Astrophysical Journal, 820(1), $22 . \quad$ doi: $10.3847 / 0004-637 \mathrm{X} / 820 / 1 / 22$

Tu, C. Y., \& Marsch, E. (1997, April). Two-Fluid Model for Heating of the Solar Corona and Acceleration of the Solar Wind by High-Frequency Alfven Waves. Solar Physics, 171 (2), 363-391. doi: 10.1023/A:1004968327196

Tu, C. Y., \& Marsch, E. (2001, May). On cyclotron wave heating and acceleration of solar wind ions in the outer corona. Journal of Geophysical Research, 106(A5), 8233-8252. doi: 10.1029/2000JA000024

Verscharen, D., Chandran, B. D. G., Jeong, S.-Y., Salem, C. S., Pulupa, M. P., \& Bale, S. D. (2019, December). Self-induced Scattering of Strahl Electrons in the Solar Wind. The Astrophysical Journal, 886(2), $136 . \quad$ doi: $10.3847 / 1538-4357 / \mathrm{ab} 4 \mathrm{c} 30$

Vocks, C., Salem, C., Lin, R. P., \& Mann, G. (2005). Electron Halo and Strahl Formation in the Solar Wind by Resonant Interaction with Whistler Waves. The Astrophysical Journal, 627(1), 540-549. Retrieved from http://stacks.iop . org $/ 0004-637 X / 627 / i=1 / a=540$ doi: $10.1086 / 430119$

Štverák, S̆., Trávníček, P., Maksimovic, M., Marsch, E., Fazakerley, A. N., \& Scime, E. E. (2008, Mar). Electron temperature anisotropy constraints in the solar wind. Journal of Geophysical Research (Space Physics), 113(A3), A03103. doi: 10.1029/2007JA012733

Wilson, I., Lynn B., Chen, L.-J., Wang, S., Schwartz, S. J., Turner, D. L., Stevens, M. L., .. Goodrich, K. A. (2019a, Dec). Electron Energy Partition across Interplanetary Shocks. II. Statistics. The Astrophysical Journal, Supplement Series, 245 (2), 24. doi: 10.3847/1538-4365/ab5445

Wilson, I., Lynn B., Chen, L.-J., Wang, S., Schwartz, S. J., Turner, D. L., Stevens, M. L., .. Goodrich, K. A. (2019b, Jul). Electron Energy Partition across Interplanetary Shocks. I. Methodology and Data Product. The Astrophysical Journal, Supplement Series, 243(1), 8. doi: 10.3847/1538-4365/ab22bd

Zouganelis, I., Maksimovic, M., Meyer-Vernet, N., Lamy, H., \& Issautier, K. (2004). A transonic collisionless model of the solar wind. The Astrophysical Journal, 606, 542-554. Retrieved from http://arxiv.org/abs/astro-ph/0402358 doi: $10.1086 / 382866$

Zouganelis, I., Meyer-Vernet, N., Landi, S., Maksimovic, M., \& Pantellini, F. (2005, 
966

967

June). Acceleration of Weakly Collisional Solar-Type Winds. The Astrophysical Journal Letters, 626(2), L117-L120. doi: 10.1086/431904 

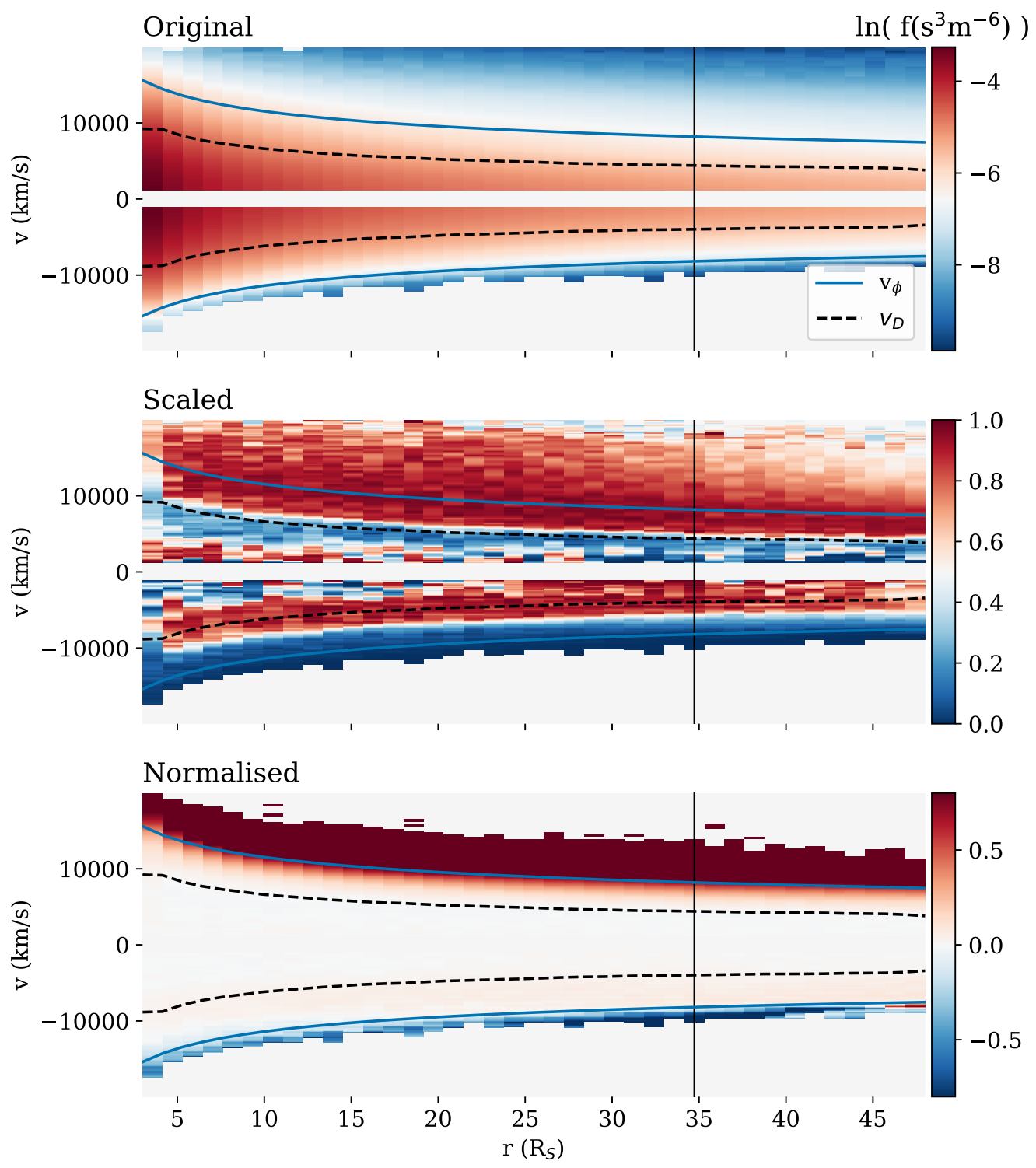

Figure A1. Parallel cuts through electron VDF plotted with respect to the radial distance in original (top), scaled (middle), and normalised (bottom) representation for the simulation run HC. $v_{\phi}$ and $v_{D}$ are marked with blue and black lines. A black vertical line denotes the radial distance of the VDFs shown in Fig. 8 (c). 

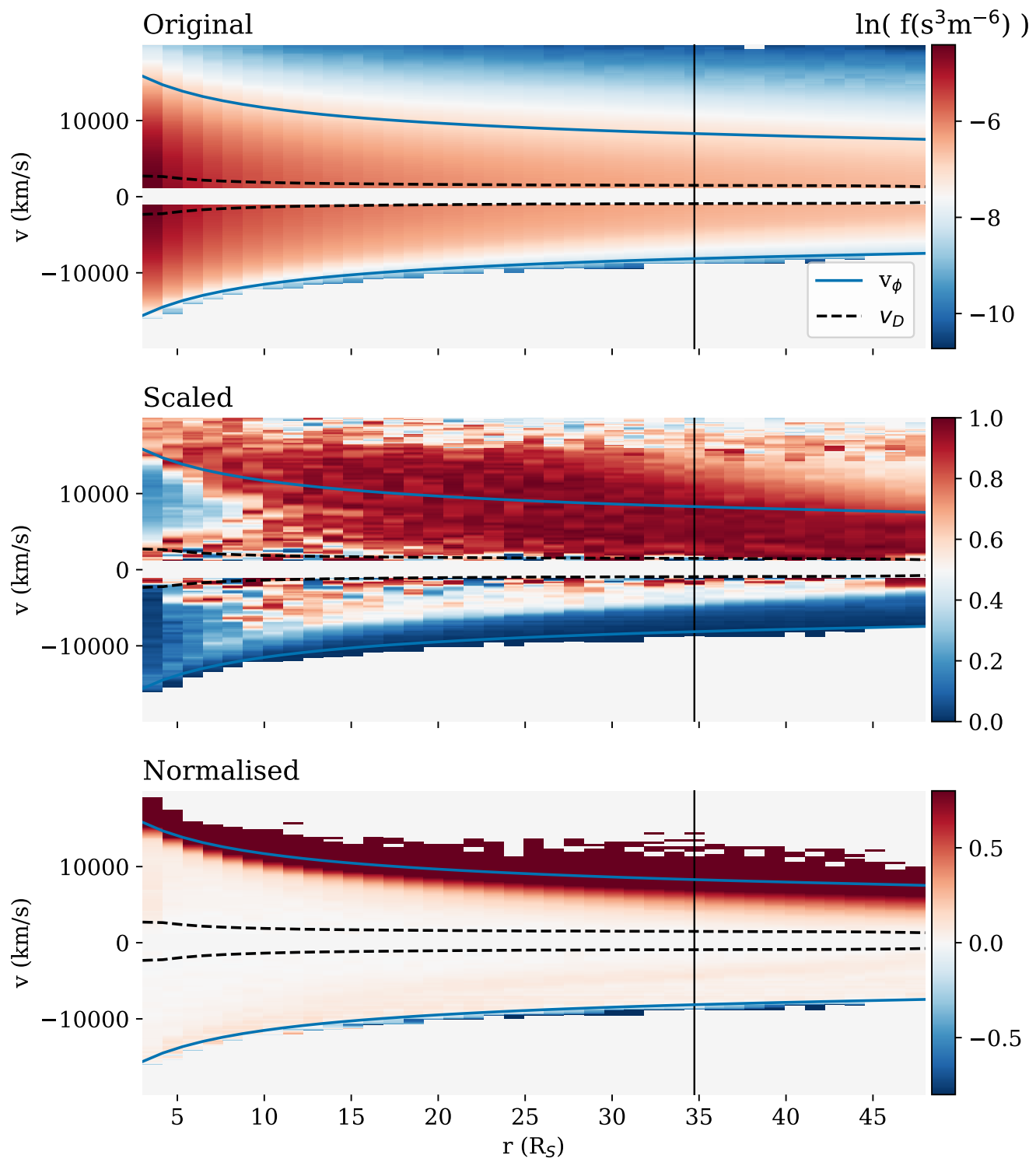

Figure A2. Parallel cuts through electron VDF plotted with respect to the radial distance in original (top), scaled (middle), and normalised (bottom) representation for the simulation run LC. $v_{\phi}$ and $v_{D}$ are marked with blue and black lines. A black vertical line denotes the radial distance of the VDFs shown in Fig. 8 (d). 

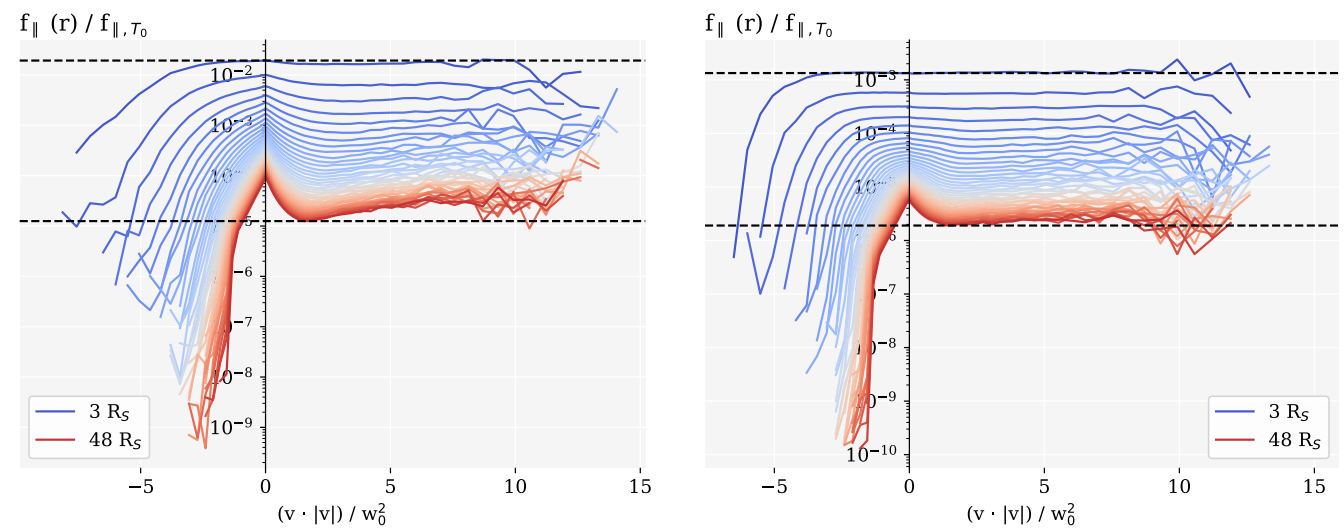

Figure B1. Electron VDFs, integrated along the $\perp$ direction $\left(f_{\|}\right)$, for different radial bins, normalised with a Maxwellian VDF with the temperature $T_{e, b o t}$. X-axis represents velocity $(v)$ multiplied with its absolute value in the units of square of thermal velocity of the electron VDF at the bottom boundary $\left(w_{0}^{2}\right)$. Radial distance is presented in colour spanning from blue closer to the Sun to red at the top boundary. Presented data is from the run HC (left) and run LC (right).
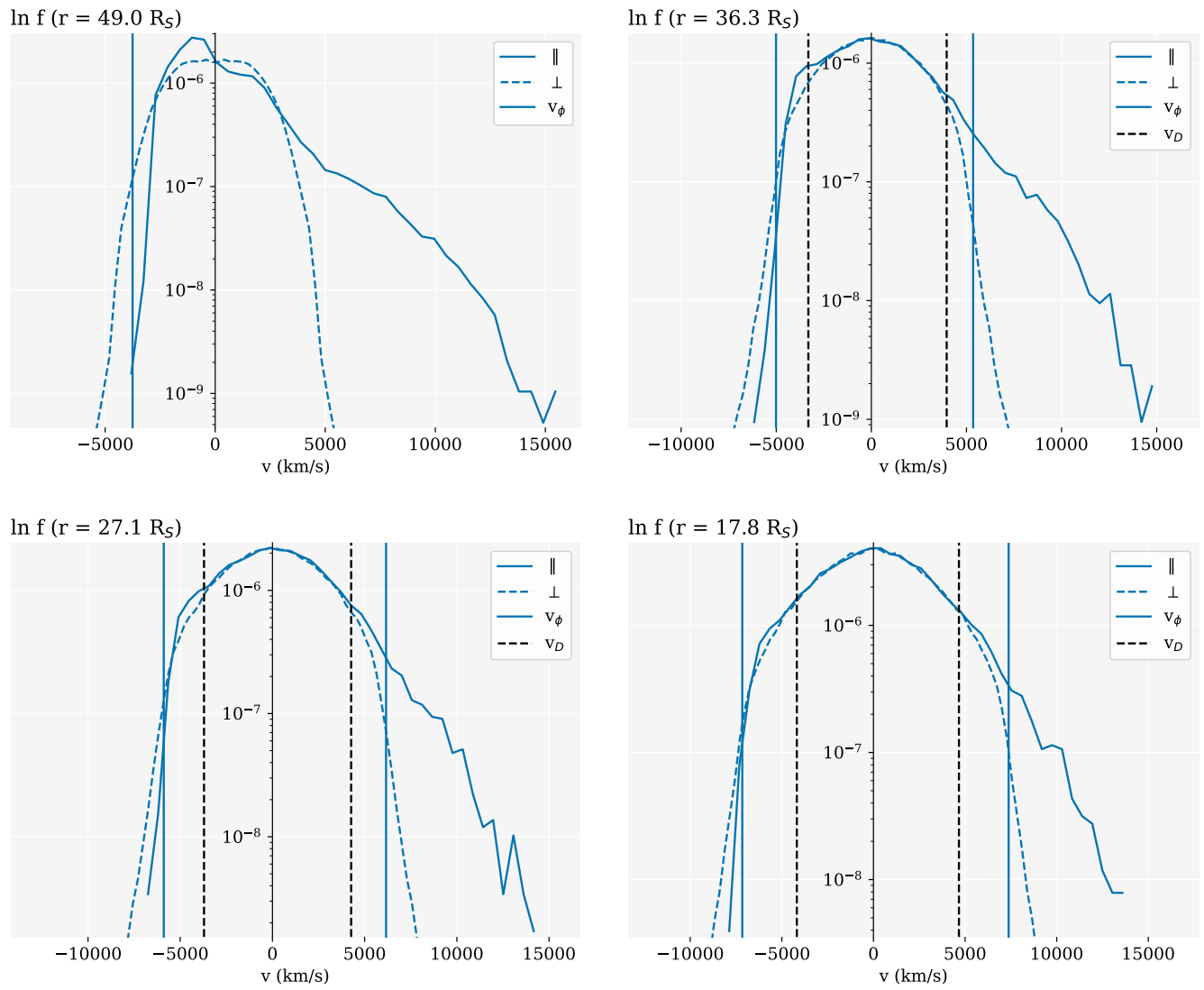

Figure C1. Parallel and perpendicular cuts through electron VDF, at different radial distances (marked in the title of each plot) for the simulation run with a non-Maxwellian top boundary condition. The electric potential velocity $\left(v_{\phi}\right)$ and the Dreicer velocity $\left(v_{D}\right)$ are marked with blue and black lines. 